\title{
Radio and X-ray study of two multi-shell supernova remnants: Kes 79 and G352.7-0.1
}

\author{
E. Giacani ${ }^{1, \star}$, M. J. S. Smith ${ }^{2}$, G. Dubner ${ }^{1, \star}$, N. Loiseau ${ }^{3}$, G. Castelletti ${ }^{1, \star}$, and S. Paron ${ }^{1, \star}$ \\ 1 Instituto de Astronomía y Física del Espacio (CONICET-UBA), CC 67, Suc. 28, 1428 Buenos Aires, Argentina \\ e-mail: egiacani@iafe.uba.ar \\ 2 XMM-Newton Science Operations Centre, ESAC/Selex I. S., Villafranca del Castillo, Spain \\ 3 XMM-Newton Science Operations Centre, ESAC/INSA, Villafranca del Castillo, Spain
}

Received 1 April 2009 / Accepted 7 July 2009

\section{ABSTRACT}

\begin{abstract}
Aims. We investigate two multi-shell galactic supernova remnants (SNRs), Kes 79, and G352.7-0.1, to understand the causes of this morphology.

Methods. The research was carried out based on new and reprocessed archival VLA observations and XMM-Newton archival data. The surrounding gas was investigated based on data extracted from the HI Canadian Galactic Plane Survey, the ${ }^{13} \mathrm{CO}$ Galactic Ring Survey, and the HI Southern Galactic Plane Survey.

Results. The present study infers that the overall morphology of both SNRs is the result of the mass-loss history of their respective progenitor stars. Kes 79 is likely to be the product of the gravitational collapse of a massive O9 star evolving near a molecular cloud and within the precursor's wind-driven bubble, while G352.7-0.1 should be the result of interactions of the SNR with an asymmetric wind from the progenitor together with projection effects. No radio point source or pulsar wind nebula was found to be associated with the X-ray pulsar CXOU J185238.6+004020 in Kes 79. The X-ray study of G352.7-0.1 found that most of the thermal X-ray radiation completely fills the interior of the remnant and originates in heated ejecta. Characteristic parameters, such as radio flux, radio spectral index, age, distance, shock velocity, initial energy, and luminosity, were estimated for both SNRs.
\end{abstract}

Key words. radio continuum: ISM - ISM: supernova remnants - X-rays: individuals: Kes 79 - X-rays: individuals: G352.7-0.1

\section{Introduction}

It is expected that between about 70 to $80 \%$ of the Galactic supernova remnants (SNRs) originate in the gravitational collapse of massive stars that end their lives with powerful supernova explosions of type Ib, Ic, and II. In these cases, it is likely that the SNRs initially expand inside the wind-driven bubble created by the progenitor stars and as a result their evolution differs considerably from that predicted for an unperturbed surrounding medium. The presence of forward and reflected shocks as well as peculiar density gradients determine the structure, shape, and duration of the successive evolutionary stages of these SNRs.

Several analytical and numerical studies have been devoted to the exploration of the interaction between supernova $(\mathrm{SN})$ ejecta and wind-driven shells (e.g., Franco et al. 1991, and references therein; Dwarkadas 2005). SN 1987A is probably the clearest observational example of the influence of the past history of the exploding star in shaping the observed remnant.

It has been suggested that the multi-shell appearance observed in the SNRs Cygnus Loop and 3C 400.2, for example, can be explained as the product of a $\mathrm{SN}$ explosion within a winddriven bubble (Gvaramadze 2006; and Velázquez et al. 2001, respectively for the Cygnus Loop and 3C 400.2 cases). In the case of Cas A, Borkowski et al. (1996) modeled X-ray emission originating in a SN explosion within a wind-blown cavity, and Reynoso et al. (1997) discovered the cold envelope of this cavity with HI absorption measurements. The multi-shell morphology can also result from the proper motion of the precursor

\footnotetext{
* Carrera del Investigador Científico of CONICET, Argentina.
}

massive star that may cause the star to explode far from the geometric center of its wind bubble, as proposed by Gvaramadze (2006). Alternative scenarios can be the existence of multiple shocks after the encounter of the blast wave with a density jump in the surrounding medium, or from simple projection effects either from three-dimensional structures of the same SNR or from multiple SNRs along the line of sight (as proposed by Uyaniker et al. 2002, to explain the morphology of Cygnus Loop). In any case, the detailed observational study of multi-shell SNRs provides insight into evolutionary and structural theories of SNRs.

To carry out a multiwavelength imaging and spectral study of this phenomenon, we selected two Galactic SNRs, Kes 79 (G33.6+0.1) and G352.7-0.1, that exhibit clear multi-shell radio structures and also have X-ray emission detected in their interiors. The present research is complemented by the study of the surrounding interstellar gas to investigate the presence of density inhomogeneities in the interstellar medium (ISM) that may have affected the expansion of the SNR shocks.

\section{The selected sources}

Kes 79: This is a Galactic SNR located at a distance of $7.1 \mathrm{kpc}$ (Case \& Bhattacharya 1998). At $1.5 \mathrm{GHz}$ and $5 \mathrm{GHz}$, it appears to consist of two concentric incomplete shells with several short and bright filaments in their interior (Velusamy et al. 1991). Based on Chandra data, Sun et al. (2004) describe the associated X-ray emission as rich in spatial structures, including filaments, three partial shells, and other features named by the authors as the "loop" and the "protrusion". Most of the X-rays 
come from the central region. In addition, Chandra observations of Kes 79 in the $0.8-8 \mathrm{keV}$ energy range detected the compact X-ray source CXOU J185238.6+004020, located close to the geometric center of the SNR (Seward et al. 2003). Later XMM-Newton observations in the $0.3-10 \mathrm{keV}$ energy band detected pulsations from CXOU J185238.6+004020 with a period of $105 \mathrm{~ms}$ (Gotthelf et al. 2005; Halpern et al. 2007). This source is likely to be the compact stellar remnant formed in the supernova event, although no evidence of a surrounding pulsar wind nebula $(\mathrm{PWN})$ was found in X-rays.

It was suggested that Kes 79 is interacting with a molecular cloud that partially surrounds the east and southeast borders of the SNR (Scoville et al. 1987; Green \& Dewdney 1992). Green \& Dewdney (1992) reported the detection of bright $\mathrm{HCO}^{+}$ $J=1-0$ emission from the eastern portion of the remnant at radial velocities close to $+105 \mathrm{~km} \mathrm{~s}^{-1}$. Taking into account the spatial and kinematical correspondences, the authors suggested that this emission emanates from material associated with the adjacent molecular cloud that has been shocked by the SNR. Further evidence of this interaction was provided by the observation of a broad and faint absorption feature seen in the $\mathrm{OH}$ spectrum between +95 and $+115 \mathrm{~km} \mathrm{~s}^{-1}$ (Green 1989).

G352.7-0.1: This SNR was classified as shell-like type, with a size of $8^{\prime} \times 6^{\prime}$ and a global spectral index of $\alpha \sim-0.6\left(S \propto v^{\alpha}\right)$ (Green 2009). A VLA image of G352.7-0.1 obtained at $1.4 \mathrm{GHz}$ with an angular resolution of 34" (Dubner et al. 1993) showed the presence of two concentric ring structures and a conspicuous unresolved bright spot over the eastern limb, whose origin was unclear. This bright source was later resolved by higher angular resolution observations and catalogued in the "New Catalog of Compact $6 \mathrm{~cm}$ Sources in the Galactic Plane" (White et al. 2005) as two separate point-like sources, WBH2005 352.775-0.153 and WBH2005 352.772-0.149. However, their connection with the SNR has yet to be clearly established.

Kinugasa et al. (1998) presented an ASCA X-ray image of G352.7-0.1 describing the emission as a shell that roughly coincides with the inner radio shell. The X-ray spectrum shows prominent K-shell lines from highly ionized Si, S, and Ar, interpreted as emission originating in shock-heated optically thin hot plasma. Kinugasa et al. (1998) proposed that G352.7-0.1 is a middle-age (2200 years old) SNR located at $8.5 \mathrm{kpc}$, evolving within a pre-existing cavity created by the stellar wind of the progenitor.

In what follows, we present new and reprocessed images in radio and in X-rays of these two SNRs, in an attempt to understand the origin of the observed characteristics.

\section{New radio and X-ray data}

\subsection{Radio data}

Kes 79 was observed as part of a high resolution study of the neighboring SNR W44, at 74 and $324 \mathrm{MHz}$ using the VLA ${ }^{1}$ in the B configuration on June 15, 2002 and in the A configuration in two sessions on August 31 and September 1, 2003. Additional observations at $324 \mathrm{MHz}$ were performed in the $\mathrm{C}$ and D arrays on December 14, 2002 and February 27, 2003, respectively. Details of the observations and reduction procedures are described in Castelletti et al. (2007). Since Kes 79 is far from the phase center of these observations, the images at both frequencies were corrected for the primary beam attenuation. The

1 The Very Large Array of the National Radio Astronomy Observatory is a facility of the National Science Foundation operated under cooperative agreement by Associated Universities, Inc.
Table 1. Observational and derived parameters of the radio data.

\begin{tabular}{ccccc}
\hline \hline $\begin{array}{c}\text { Frequency } \\
(\mathrm{MHz})\end{array}$ & $\begin{array}{c}\text { Beam } \\
\left({ }^{\prime \prime} \times\right)\end{array}$ & $\begin{array}{c}\text { PA } \\
\circ\end{array}$ & $\begin{array}{c}\text { Noise } \\
\left(\mathrm{mJy} \mathrm{beam}^{-1}\right)\end{array}$ & $\begin{array}{c}\text { Flux density } \\
(\mathrm{Jy})\end{array}$ \\
\hline 74 & $39.0 \times 36.0$ & Kes 79 & 60 & $76 \pm 10$ \\
324 & $13.3 \times 13.2$ & -47 & 5.8 & $39 \pm 8$ \\
1500 & $18.6 \times 17.0$ & -65.12 & 1.24 & $1.0 \pm 1.5$ \\
4800 & $14.5 \times 13.0$ & -84.8 & 0.34 & - \\
\hline \multicolumn{5}{c}{$\mathbf{G 3 5 2 . 7 - 0 . 1}$} \\
1400 & $37.0 \times 33.0$ & 18.33 & 1 & $3.1 \pm 0.3$ \\
4800 & $12.0 \times 9.0$ & -41.34 & 0.2 & - \\
\hline
\end{tabular}

synthesized beam, rms noise level and other observational parameters are listed in Table 1. It is important to remark that the images at both frequencies are sensitive to all spatial scales because the size of Kes $79\left(\sim 10^{\prime}\right)$ is smaller than the largest well imaged structures at the respective frequencies $\left(\sim 36^{\prime}\right.$ at $74 \mathrm{MHz}$ and $\sim 70^{\prime}$ at $324 \mathrm{MHz}$ ).

We also reprocessed VLA archival data obtained in the direction of Kes 79 at $1.5 \mathrm{GHz}$. The observations were taken on July 4, 1989 (BnC configuration, project code AV166), and on December 24, 1989 (D configuration, project code AV165). The interferometric data were combined in the $u v$ plane with singledish information extracted from the Effelsberg $100 \mathrm{~m} 1.4 \mathrm{GHz}$ Survey (Reich et al. 1990) to recover structures at all spatial frequencies.

To investigate in detail the radio emission close to the X-ray pulsar candidate while searching for a radio pulsar wind nebula, we also reprocessed $4.8 \mathrm{GHz}$ data corresponding to observations carried out with the VLA in the D array in 1984, September 8 (Project code $\mathrm{AD} 131$ ) and in the $\mathrm{D}$ and $\mathrm{BnC}$ configurations in 1989, July 3 and 4, respectively (Project code AV165 and AV166, respectively). These data are only useful for the study of structures smaller than $\sim 5^{\prime}$, which correspond to the largest angular scale that can be imaged reasonably well with the array at this frequency. Since no single dish data at $4.8 \mathrm{GHz}$ are publicly available for this region, the largest scale structures have not been fully recovered and therefore no attempt to estimate the total flux density is made at this frequency.

For the SNR G352.7-0.1 we reprocessed VLA archival data at 1.4 and $4.8 \mathrm{GHz}$. The observations at $1.4 \mathrm{GHz}$ were performed in the CnD array on February 23, 1991 (project AD260). The image at $4.8 \mathrm{GHz}$ was produced from the observations carried out in the DnC configuration on November 3, 1985 (project AH206). Observational parameters are also included in Table 1. As in the case of Kes 79, we did not derive the total flux density of this SNR at $4.8 \mathrm{GHz}$ because the largest angular scale contributions are missing.

\subsection{X-ray data}

For Kes 79, we analyzed two XMM-Newton observations, 0204970201 and 0204970301, which were performed on October, 17-18 2004 and on October, 23-24 2004, respectively, with a $30 \mathrm{ks}$ duration each. In both observations, the MOS cameras were operated in full frame mode, with a medium filter. The complete extent of the SNR was included in the MOS fields of view. As the PN camera was operated in small window mode only part of the remnant was detected, and hence we did not include these data in our analysis.

In the case of G352.7-0.1, we analyzed the XMM-Newton observation 0150220101 which was performed on October 3, 
Table 2. Integrated flux densities on the SNR Kes79.

\begin{tabular}{lclccl}
\hline \hline $\begin{array}{l}\text { Frequency } \\
(\mathrm{MHz})\end{array}$ & $\begin{array}{c}\text { Scaled flux } \\
\text { density }(\mathrm{Jy})\end{array}$ & References & $\begin{array}{l}\text { Frequency } \\
(\mathrm{MHz})\end{array}$ & $\begin{array}{l}\text { Scaled flux } \\
\text { density (Jy) }\end{array}$ & References \\
\hline $30.9 \ldots \ldots$ & $94 \pm 19^{(a)}$ & Kassim (1989) & $408 \ldots \ldots$ & $44.0 \pm 7.0$ & Kesteven (1968) \\
$74 \ldots \ldots$ & $76 \pm 10^{(b)}$ & This work & $430 \ldots \ldots$ & $34 \pm 10^{(c)}$ & Kundu \& Velusamy (1967) \\
$80 \ldots \ldots$ & $101 \pm 23^{(a)}$ & Dickel (1973) & $1410 \ldots \ldots$ & $9.0 \pm 3.0$ & Beard \& Kerr (1969) \\
$80 \ldots \ldots$ & $54 \pm 8^{(a)}$ & Slee (1977) & $1414 \ldots \ldots$ & $13.0 \pm 0.4$ & Altenhoff et al. (1970) \\
$80 \ldots \ldots$ & $70 \pm 20^{(a)}$ & Dulk \& Slee (1972) & $1415 \ldots \ldots$ & $20.0 \pm 6.0$ & Caswell et al. (1981) \\
$83 \ldots \ldots$ & $76 \pm 12^{(a)}$ & Kovalenko et al. (1994) & $1500 \ldots \ldots$ & $11.5 \pm 1.5^{(b)}$ & This work \\
$111 \ldots \ldots$ & $64 \pm 12^{(a)}$ & Kovalenko et al. (1994) & $2650 \ldots \ldots$ & $8.0 \pm 2.0$ & Beard \& Kerr (1969) \\
$160 \ldots \ldots$ & $57 \pm 17^{(a)}$ & Slee (1977) & $2695 \ldots \ldots$ & $9.0 \pm 0.4$ & Altenhoff et al. (1970) \\
$324 \ldots \ldots$ & $39 \pm 8^{(b)}$ & This work & $5000 \ldots \ldots$ & $5.2 \pm 0.4$ & Altenhoff et al. (1970) \\
$330 \ldots \ldots$ & $35 \pm 7^{(a)}$ & Kassim (1992) & $5000 \ldots \ldots$ & $7.8 \pm 0.8$ & Caswell et al. (1975) \\
$408 \ldots \ldots$ & $34.4 \pm 3.4$ & Caswell et al. (1975) & $10600 \ldots$ & $6.7 \pm 1.5$ & Becker \& Kundu (1975) \\
\hline
\end{tabular}

${ }^{(a)}$ No correction to Baars et al. (1977) scale was applied. ${ }^{(b)}$ Flux density scale from VLA Calibrator Manual,

http:/www.aoc.nrao.edu/ gtaylor/calib.html. ${ }^{(c)}$ The correction factor was not available.

2002. Both MOS and PN cameras were operated in full frame mode with a medium filter. The net exposure times were about $25 \mathrm{ks}$ and $20 \mathrm{ks}$ for the MOS and PN cameras, respectively.

For both remnants the raw event files were obtained from the XMM-Newton Science Archive. The data were processed with the Science Analysis System (SAS) version 8.0 and the most upto-date calibration files. The astrometry of the resulting images was confirmed to be accurate to about $5^{\prime \prime}$.

\section{Results for Kes 79}

\subsection{Radio emission distribution}

We present the first radio image of Kes 79 at $74 \mathrm{MHz}$ and the highest angular resolution image ever obtained at $324 \mathrm{MHz}$ (Fig. 1, upper panels). Figure 1 also includes the reprocessed image at $1.5 \mathrm{GHz}$ (lower left panel), which represents an improvement with respect to the previously published one because with the addition of single-dish data all spatial frequencies are present. In Fig. 1 (lower right panel), we have also included for comparison the XMM-Newton image obtained in the energy range $(0.5-5) \mathrm{keV}$.

The new low-frequency radio images show the same double shell structure previously noticed by Velusamy et al. (1991) at higher frequencies. The outer radio shell, about $10^{\prime}$ in size, is circularly symmetric along the SW and W borders of the remnant, while towards the NE and $\mathrm{E}$ the shell has a protrusion close to Dec $\sim 0^{\circ} 44^{\prime}$ that inverts the shape into a concave curvature. The inner radio shell, about 6'.5 in size, is brighter than the outer one at all inspected radio frequencies. It looks incomplete and fragmented. Towards the $S$, this shell consists of a series of short arc-like filaments, while towards the $\mathrm{E}$ it is fainter and rather straight.

The high angular resolution and sensitivity achieved at $324 \mathrm{MHz}$ shows the presence of a small cometary-like feature, about $60^{\prime \prime} \times 40^{\prime \prime}$ in size, near the geometric center of the remnant, the X-ray compact source CXOU J185238.6+004020 lying at the southern border of this structure, whose maximum is about $10^{\prime \prime}$ north. We note that the morphology of this nebula is similar to that of some radio pulsar wind nebula (PWN), motivating us to develop future investigations (see Sect. 4.2).

On the basis of the new images, we estimated the flux density at 74, 324, and $1500 \mathrm{MHz}$. The results are listed in Table 1 together with the observational parameters. The errors quoted in the flux density estimates include the rms noise of each image and uncertainties in the determination of the boundaries of the SNR emission. We note that the total integrated flux density estimated at $1.5 \mathrm{GHz}$ based on the interferometric observations, agrees within the errors with earlier estimates obtained from single-dish observations and presented by Beard \& Kerr (1969) $\left(S_{1410 \mathrm{MHz}}=9 \pm 3 \mathrm{Jy}\right)$ and Altenhoff et al. (1970) $\left(S_{1414 \mathrm{MHz}}=13 \pm 0.4 \mathrm{Jy}\right)$. This confirms the accuracy of the flux density estimate and the spectral study discussed in the next section.

\subsection{Radio spectral index}

We carried out a study of the global radio continuum spectrum of Kes 79, based on our low radio frequencies images at 74 and $324 \mathrm{MHz}$ and the reprocessed $1.5 \mathrm{GHz}$ data together with those taken from the literature. Table 2 lists the values used to fit the global radio spectrum. We do not include flux densities obtained from either observations made with instruments having insufficient resolution, or those in strong disagreement with other measurements made at nearby frequencies. Most of the measurements for frequencies above $408 \mathrm{MHz}$ were brought to the absolute flux density scale of Baars et al. (1977). In some cases, an estimate of the correction factor was unavailable because the original reference did not list the assumed flux densities of the primary calibrators. Below $408 \mathrm{MHz}$, where the systematic error of the scale of Baars et al. (1977) is more than 5\%, no scaling was applied. These values have nevertheless been included in our fit because they do not exhibit a large spread.

The radio spectrum of SNR Kes 79 traced from all frequencies listed in Table 2 follows a single power law $S_{v} \propto v^{\alpha}$ across four orders of magnitude in frequency, with an index $\alpha=-0.58 \pm 0.03$, and the fitting is shown in Fig. 2 .

We also searched for variations in the spectral index across the remnant by comparing the brightness distribution at the different observed frequencies. To study the spectral variations as a function of position within the remnant, we produced multifrequency images of identical beam size and shape. For our study, the $u v$ data at $324 \mathrm{MHz}$ were tapered to match the synthesized beam of the data at 74 and $1500 \mathrm{MHz}$. The images were also aligned and interpolated to identical projections (e.g., field center, pixel separation).

Following the same behavior noted above for the global spectral index, which is constant over several decades in frequency, we find that the spatial distribution of the radio spectrum between 74 and $324 \mathrm{MHz}$, is morphologically similar to the spectral map traced between 324 and 1500 MHz. In Fig. 3, 

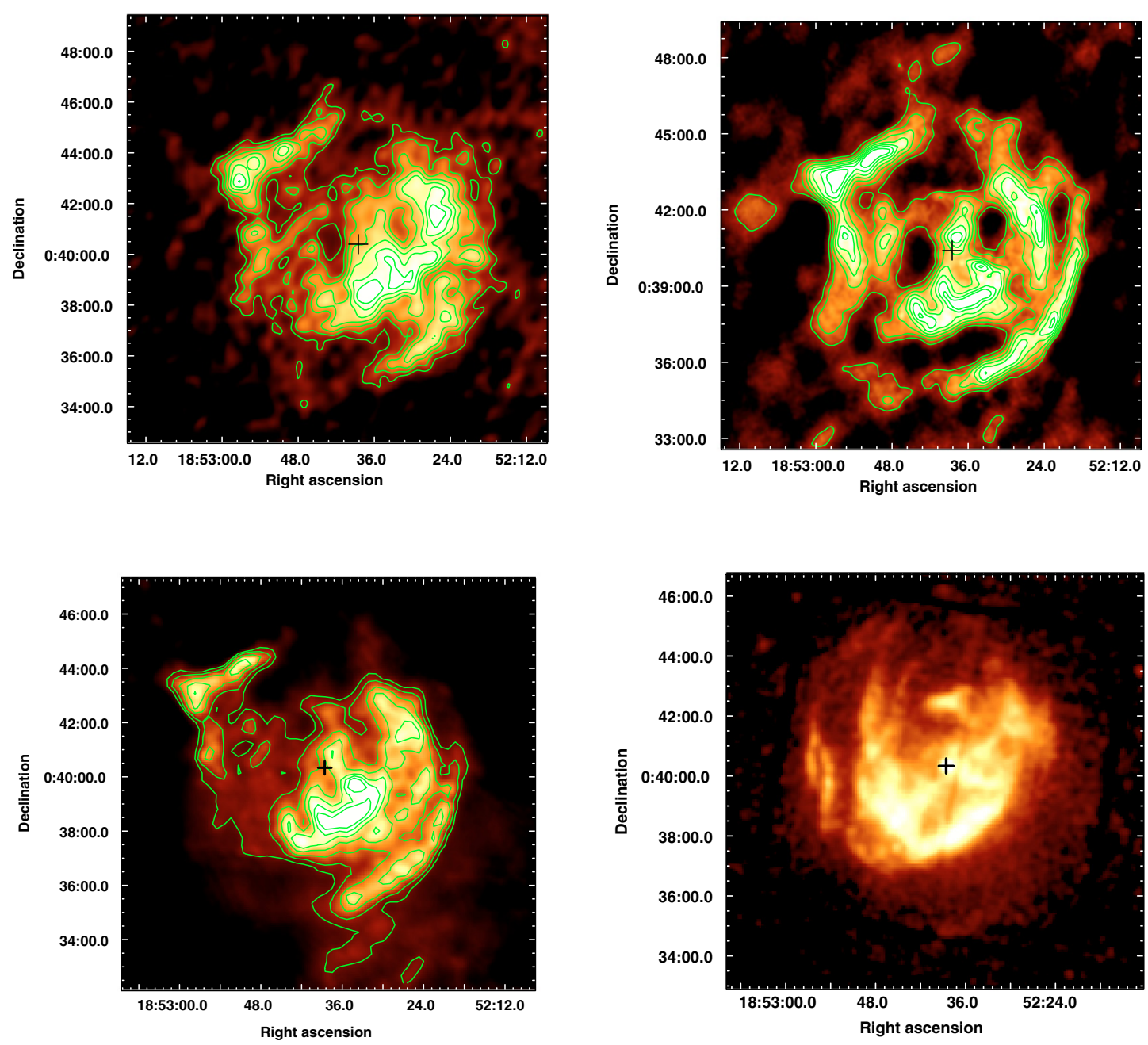

Fig. 1. Upper left: VLA image of Kes 79 at $74 \mathrm{MHz}$. The synthesized beam is $39^{\prime \prime} \times 36^{\prime \prime}$ and the rms noise is $60 \mathrm{mJy}^{-1}$ beam $^{-1}$. Plotted contours go from 0.2 to $0.8 \mathrm{mJy}_{\text {beam }}{ }^{-1}$ in steps of $0.1 \mathrm{mJy}^{-1} \mathrm{bem}^{-1}$. Upper right: the same at $324 \mathrm{MHz}$. The synthesized beam is $13^{\prime \prime} \times 13^{\prime \prime}$ and the rms noise is $5.8 \mathrm{mJy}_{\text {beam }}^{-1}$. Plotted contours go from 14 to $50 \mathrm{mJy} \mathrm{beam}^{-1}$, in steps of $6 \mathrm{mJy} \mathrm{beam}^{-1}$. Lower left: the same at $1.5 \mathrm{GHz}$. Single dish data from Effelsberg $100 \mathrm{~m}$ telescope have been added. The angular resolution is $19^{\prime \prime} \times 17^{\prime \prime}$ and the rms noise $1.2 \mathrm{mJy}^{-1}$ beam ${ }^{-1}$. Plotted contours are 10 , 12, $16,22,28,34,40$, and $46 \mathrm{mJy}^{\text {beam }}{ }^{-1}$. Lower right: XMM-Newton image of Kes 79 in the energy range $0.5-5 \mathrm{keV}$. It has been square-root scaled to emphasize faint emission. The plus sign included in all panels indicates the position of the point-like X-ray source CXOU J185238.6+004020.

we show this last comparison degraded to an angular resolution of $40^{\prime \prime}$ to remove unphysical small scale fluctuations. In the comparison, the respective signals of each radio image were clipped at a $4 \sigma$ rms noise level. In Fig. 3, dark areas correspond to a steep spectrum $(\alpha \sim-0.7)$, while light gray corresponds to flatter indices $(\alpha \sim-0.4)$. The uncertainties vary between 0.04 and 0.1 depending on the intensity of the emission compared. A few contours of the radio intensity at $324 \mathrm{MHz}$ are superimposed for reference. The radio spectral index distribution is practically featureless, with little departure from the global spectral index $(\alpha \sim-0.6)$. Darker regions observed close to the limb of the SNR are probably caused by the larger uncertainties in regions of low emission at $1.5 \mathrm{GHz}$.

We also conducted a careful study of the radio spectrum close to the small nebulosity identified in the image at $324 \mathrm{MHz}$ as a possible PWN candidate. If this is the nebula created by fresh particles and magnetic fields injected by the pulsar detected in X-rays (shown with a plus sign in Fig. 3), a flat radio spectrum with $\alpha$ between $\sim-0.3$ and 0 is expected for it (Gaensler $\&$ Slane 2006). To carry out this search, we used our data at $324 \mathrm{MHz}$ and the reprocessed archival data at 1.5 and $4.8 \mathrm{GHz}$. As mentioned in Sect. 3.1, at $4.8 \mathrm{GHz}$ the largest scale structures have not been fully recovered, but the flux density estimate for this small structure is reliable because the size of the studied feature is smaller than the largest well imaged structure at $4.8 \mathrm{GHz}$ $\left(5^{\prime}\right)$. We obtained a total flux density for the nebular emission of $0.26 \pm 0.10 \mathrm{Jy}, 0.11 \pm 0.03 \mathrm{Jy}$, and $0.05 \pm 0.06 \mathrm{Jy}$ at 324,1500 , and $4800 \mathrm{MHz}$, respectively. The errors quoted include the rms noise of each image and the uncertainty in the choice of the integration boundaries. We derive a radio spectral index $\alpha \simeq-0.6$ between 324 and $4800 \mathrm{MHz}$. This steep value, compatible with the global spectral index of Kes 79, rules out the possibility that this feature is the radio PWN.

In summary, the detailed radio spectral study carried out for the first time in the SNR Kes79 allows us to conclude that both inner and outer radio shells have similar spectral properties and no traces of radio PWN are found near the point X-ray source CXOU J185238.6+004020.

\subsection{The environs of Kes 79}

We investigated the distribution of the interstellar gas in the surroundings of Kes 79 . We mapped the neutral gas in its vicinity to search for morphological and kinematical signatures of atomic 


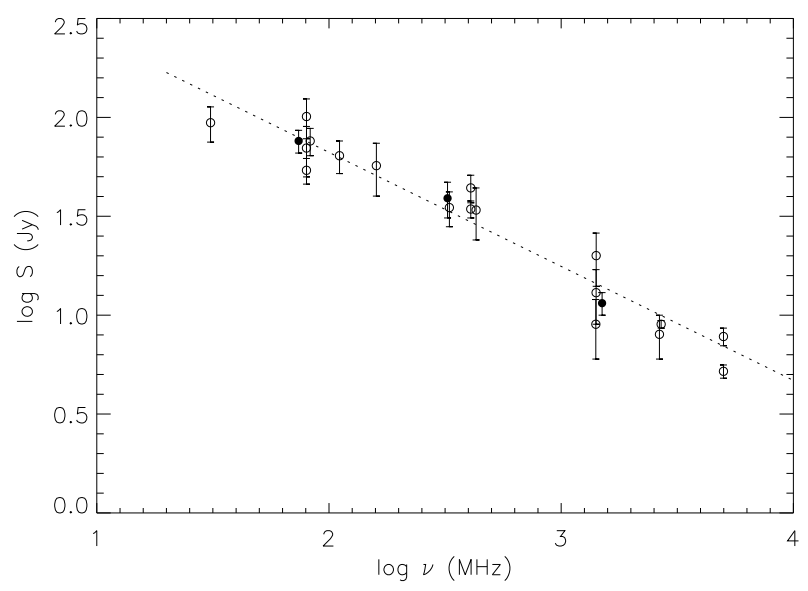

Fig. 2. Radio spectrum of the SNR Kes 79 obtained from the flux density values listed in Table 2 . The filled circles correspond to data from the new VLA measurements at 74 and $324 \mathrm{MHz}$, and the reprocessed image at $1500 \mathrm{MHz}$, while the rest of the values were taken from the literature and homogenized. The dashed line represents the best fit linear function to the data points, which yields a global spectrum for the remnant $\alpha=-0.58 \pm 0.03$.

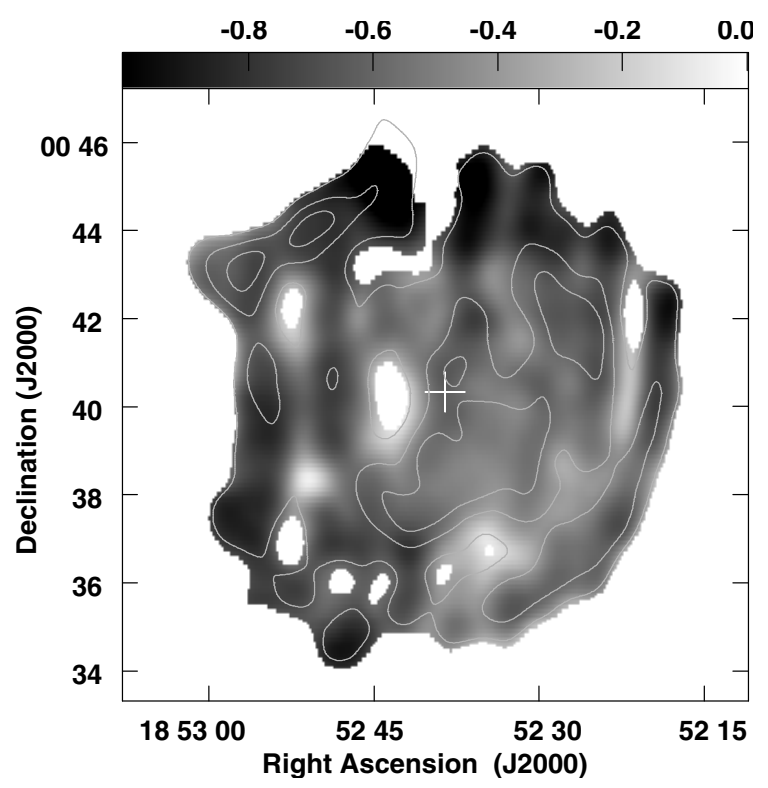

Fig. 3. Distribution of the spectral index over Kes 79 between 324 and $1500 \mathrm{MHz}$, as computed from images made with a matched restoring beam of $40^{\prime \prime}$. For reference, the $324 \mathrm{MHz}$ contours at 0.1 , $0.2,0.3$ and $0.5 \mathrm{Jy}$ beam $^{-1}$ are plotted. The position of the CXOU J185238.6+004020 source is indicated by a white plus sign. Regions with flux density below $4 \sigma$ were clipped.

material associated with the SNR. The HI data were extracted from the Canadian Galactic Plane Survey (CGPS, see Taylor et al. 2003, for details). The final data have an angular resolution of about $1^{\prime}$ and the velocity resolution is $1.3 \mathrm{~km} \mathrm{~s}^{-1}$ with a channel separation of $0.82 \mathrm{~km} \mathrm{~s}^{-1}$.

After careful inspection of the entire HI cube, we find that the only channels with an indication of possible physical association are limited to the velocity range between +90 and $+99 \mathrm{~km} \mathrm{~s}^{-1}$ (all velocities are referred to the local standard of rest). Figure 4 shows the HI distribution averaged over the aforementioned velocity interval, with some contours of Kes 79 at $324 \mathrm{MHz}$ superimposed for reference. In this figure, an open HI shell with a radius of about 8.5 , centered close to $18^{\mathrm{h}} 52^{\mathrm{m}} 36.6^{\mathrm{s}}, 00^{\circ} 41^{\prime} 02^{\prime \prime}$

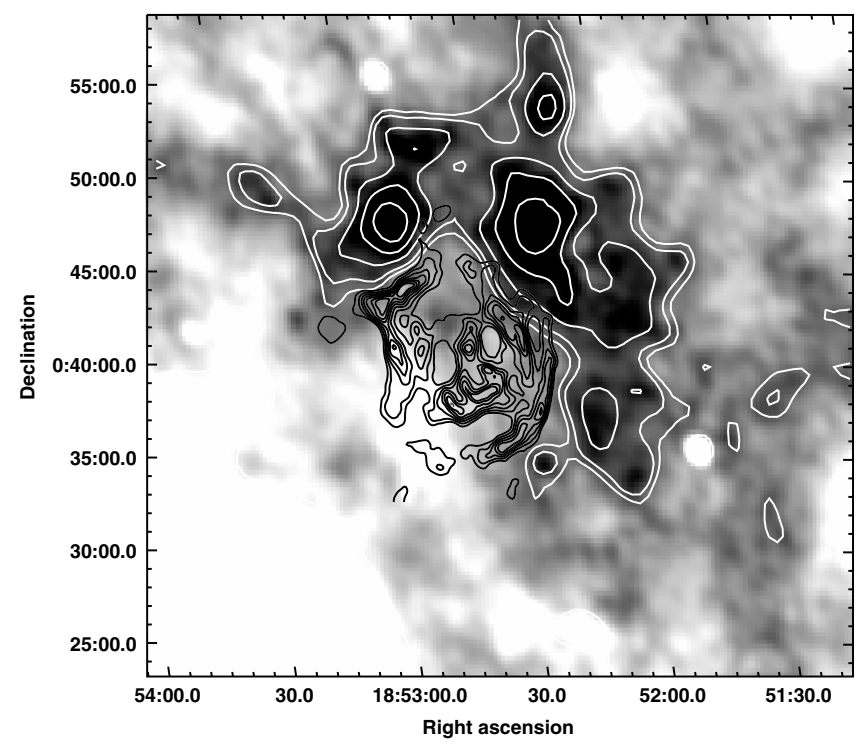

Fig. 4. Grayscale and contours image of the HI distribution around Kes 79 integrated between 90 and $99 \mathrm{~km} \mathrm{~s}^{-1}$. The black contours represent the $324 \mathrm{MHz}$ radio continuum emission. Grays vary between 80 and $115 \mathrm{~K}$ and the plotted contours correspond to 103, 106, 109, 113, and $118 \mathrm{~K}$.

(J2000.0), which embraces most of the periphery of Kes 79, can be identified. Interestingly, part of this shell exactly matches the shape of the radio continuum emission along the NE flattened border, close to $18^{\mathrm{h}} 53^{\mathrm{m}} 00^{\mathrm{s}}, 00^{\circ} 45^{\prime} 00^{\prime \prime}$.

If we assume that the HI gas in this velocity range is physically associated with Kes 79, we can adopt $v \sim+95 \mathrm{~km} \mathrm{~s}^{-1}$ as the systemic velocity of Kes 79. By applying the flat Galactic rotation curve of Fich et al. (1989) (with $R_{\odot}=8.5 \mathrm{kpc}$ and $V_{\odot}=220 \mathrm{~km} \mathrm{~s}^{-1}$ ), we obtain kinematic distances of either $\sim 6.5$ or $\sim 7.5 \mathrm{kpc}$. The difference between these two values is caused by the distance ambiguity in the inner part of the Galaxy and both are in good agreement with the distance of $7.1 \mathrm{kpc}$ proposed by Case \& Bhattacharya (1998). In the absence of any additional discriminator between the two kinematical distances, in what follows we adopt a distance to Kes 79 of $7 \mathrm{kpc}$.

We can derive a number of parameters characterizing the HI shell. By assuming optically thin gas and integrating the column density between +90 and $+99 \mathrm{~km} \mathrm{~s}^{-1}$, the total mass in the shell is about $3000 M_{\odot}$. A density of $\sim 8 \mathrm{~cm}^{-3}$ is calculated for the HI shell by assuming that its radius and thickness are about $17 \mathrm{pc}$ and $10 \mathrm{pc}$, respectively $\left(8.5\right.$ and $5^{\prime}$ at the adopted distance of $7 \mathrm{kpc}$ ). If this shell was formed by ISM swept up by the SNR shock, then the kinetic energy injected into the ISM is about $1.5 \times 10^{48} \mathrm{erg}$. This value was calculated by assuming an expansion velocity of $\sim 7 \mathrm{~km} \mathrm{~s}^{-1}$, as suggested from the HI data. This expansion velocity is a lower limit, since confusion from unrelated foreground and background emission impedes the detection of possible caps of the expanding shell. The estimated values have uncertainties of up to $40 \%$ caused mainly by the error in the distance, and secondarily by the choice of the background level, the expansion velocity, and the integration boundaries. The low expansion velocity found for this HI shell implies that this must be an old structure, probably created along thousand of years by the action of the precursor's stellar wind.

We also investigated the molecular gas in the surroundings of Kes 79 using observations of the ${ }^{13} \mathrm{CO} J=1-0$ line at $110 \mathrm{GHz}$. The data were extracted from the Galactic Ring Survey (Jackson et al. 2006) for which the angular and spectral resolution are 

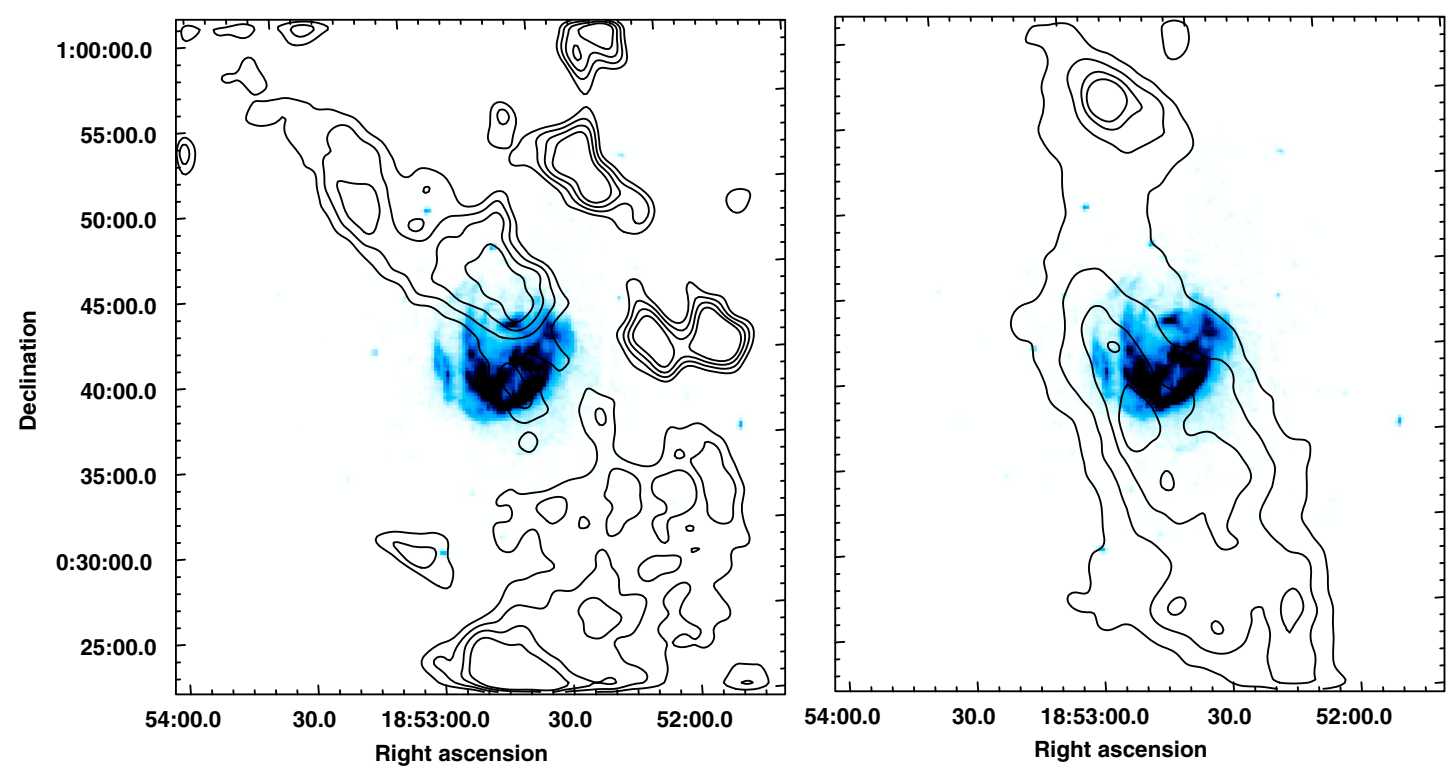

Fig. 5. Left: contours image of the ${ }^{13} \mathrm{CO} J=1-0$ line integrated between +88.0 and $+94.5 \mathrm{~km} \mathrm{~s}^{-1}$ with the XMM-Newton X-ray emission. The contours plotted are $1.9,2.3,2.8$, and $3.3 \mathrm{~K} \mathrm{~km} \mathrm{~s}^{-1}$. Right: contours image of the ${ }^{13} \mathrm{CO} J=1-0$ line integrated between +99.0 and $+109.0 \mathrm{~km} \mathrm{~s}{ }^{-1}$ with the XMM-Newton X-ray emission. The contours plotted are $3.3,4.0$ and $4.7 \mathrm{~K} \mathrm{~km} \mathrm{~s}^{-1}$.

$46^{\prime \prime}$ and $0.21 \mathrm{~km} \mathrm{~s}^{-1}$, respectively. After examining the entire data cube, we found morphological signatures of a possible interaction between the SNR and the surrounding molecular material when limited to the velocity intervals $\left(+88.0 \mathrm{~km} \mathrm{~s}^{-1}\right.$, $\left.+94.5 \mathrm{~km} \mathrm{~s}^{-1}\right)$ and $\left(+99.0 \mathrm{~km} \mathrm{~s}^{-1},+109.0 \mathrm{~km} \mathrm{~s}^{-1}\right)$. Figure 5 displays the distribution of the ${ }^{13} \mathrm{CO} J=1-0$ emission integrated from +88.0 to $+94.5 \mathrm{~km} \mathrm{~s}^{-1}$ (left) and between +99.0 and $+109 \mathrm{~km} \mathrm{~s}^{-1}$ (right) superimposed for comparison, on the XMMNewton X-ray emission.

The first obvious conclusion is that the optically thin ${ }^{13} \mathrm{CO}$ emission does not follow the same distribution as the HI. The most conspicuous molecular feature observed between +88.0 and $+94.5 \mathrm{~km} \mathrm{~s}^{-1}$ is an elongated molecular cloud towards the NE of Kes 79, whose maximum coincides with a region of faint diffuse $\mathrm{X}$-ray emission, suggesting that it is responsible for greater X-ray absorption. Between +99 and $+109 \mathrm{~km} \mathrm{~s}^{-1}$, an extended $\mathrm{CO}$ cloud is detected crossing the remnant from $\mathrm{N}$ to $\mathrm{S}$. This molecular cloud coincides with the location of earlier detections of $\mathrm{HCO}^{+}$and ${ }^{12} \mathrm{CO}$ emission, and of the faint $\mathrm{OH}$ absorption reported by Green (1989) and Green \& Dewdney (1992).

Neither of the two molecular clouds show direct kinematic evidence of interaction with Kes 79 (e.g., broadenings in the molecular spectra, asymmetries in the profiles), although the lack of these signatures of interactions is not uncommon in molecular clouds that are known to be interacting with SN shocks (e.g., as in the case of the SNR 3C 391, Wilner et al. 1998).

An estimate of the atomic and molecular gas column density between us and Kes 79 was calculated from $N_{\mathrm{H}}=2 N\left(\mathrm{H}_{2}\right)+$ $N(\mathrm{HI})$ by assuming local thermodynamic equilibrium, a uniform excitation temperature $T_{\mathrm{ex}} \sim 10 \mathrm{~K}$ for the ${ }^{13} \mathrm{CO} \mathrm{J}=1-$ 0 transition along the line of sight, and a calibration ratio of $N\left(\mathrm{H}_{2}\right) / N\left({ }^{13} \mathrm{CO}\right)=5 \times 10^{5}$ (Dickman 1978). The hydrogen column density obtained, $\sim 2.2 \times 10^{22} \mathrm{~cm}^{-2}$, is compatible within errors with the absorbing column density estimated from the spectral fit of Chandra and XMM-Newton data for the entire remnant $\left(\sim 1.6 \times 10^{22} \mathrm{~cm}^{-2}\right.$, Sun et al. 2004 and $\sim 1.5 \times 10^{22} \mathrm{~cm}^{-2}$, this work).

\subsection{Analysis of the X-ray emission and comparison with radio}

Figure 6 shows the detailed comparison between radio at $324 \mathrm{MHz}$ and X-ray emission features. It can be seen that the most intense and structured X-ray emission is confined to a circular region within the perimeter of the inner radio shell, while fainter and diffuse X-ray emission extends towards the outer radio limb. As noted before by Sun et al. (2004), an interesting correspondence can be observed between both type of emission, although the agreement is not exact. The best radio/X-ray matching occurs predominantly along the southern border of the inner radio shell, where one of the two curved bright radio filaments visible near RA $\sim 18^{\mathrm{h}} 52^{\mathrm{m}} 42^{\mathrm{s}}$, Dec $\sim 0^{\circ} 38^{\prime}$, has an exact spatial correlation with bright X-ray emission. The second almost parallel radio filament in the region, however, does not have an X-ray counterpart. In the same inner south limb, the X-ray feature called the "loop" by Sun et al. (2004) (near $18^{\mathrm{h}} 52^{\mathrm{m}} 50^{\mathrm{s}}, 00^{\circ} 37^{\prime}$ ), has a good correspondence with a faint radio feature. Towards the E, on the other hand, bright twisted X-ray filaments, which were suggested by Sun et al. (2004) to be thin shells viewed edge-on, are almost exactly correlated with the shape of the radio emission along the outer shell. Another peculiarity worth noting is that the eastern border of the inner shell appears to be almost straight in both spectral regimes. In addition, the flat radio border of Kes 79 near the northern border of the inner shell, around $18^{\mathrm{h}} 52^{\mathrm{m}} 38^{\mathrm{s}}, 00^{\circ} 42^{\prime} 24^{\prime \prime}$, also corresponds with an X-ray bright feature.

Narrow-band images of Kes 79 were produced covering the emissions of $\mathrm{Ne}(0.85-1.25 \mathrm{keV}), \mathrm{Mg}(1.30-1.50 \mathrm{keV}), \mathrm{Si}$ (1.70-2.20 keV), and S (2.35-2.55 keV) (Fig. 7). The same gray scale is used in all four images to facilitate the comparison of data for the respective bands. The central emission is quite similar across the four bands and to the broadband emission displayed in Figs. 1 and 6, with the only exception of sulfur, which is notably weaker than the rest. From Fig. 7, it can be seen that the $\mathrm{Mg}$ and especially the $\mathrm{Si}$ band emission are more prominent in the northwest of the inner shell than that of the Ne band, 

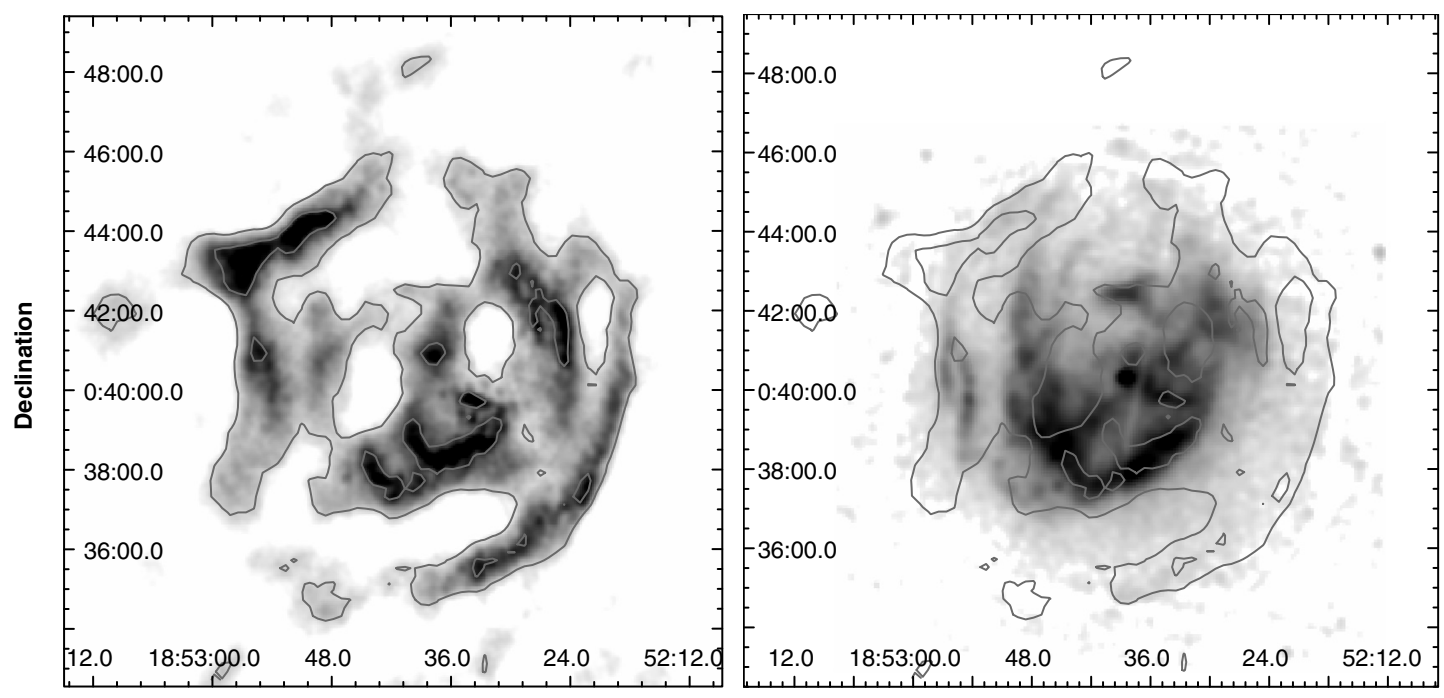

Right ascension

Fig. 6. Comparison between the radio emission of Kes 79 at $324 \mathrm{MHz}$ (left panel) and the XMM Newton X-ray image obtained between 0.5 and $5 \mathrm{KeV}$ (right panel). Some radio contours overlap the X-ray image to facilitate the comparison.
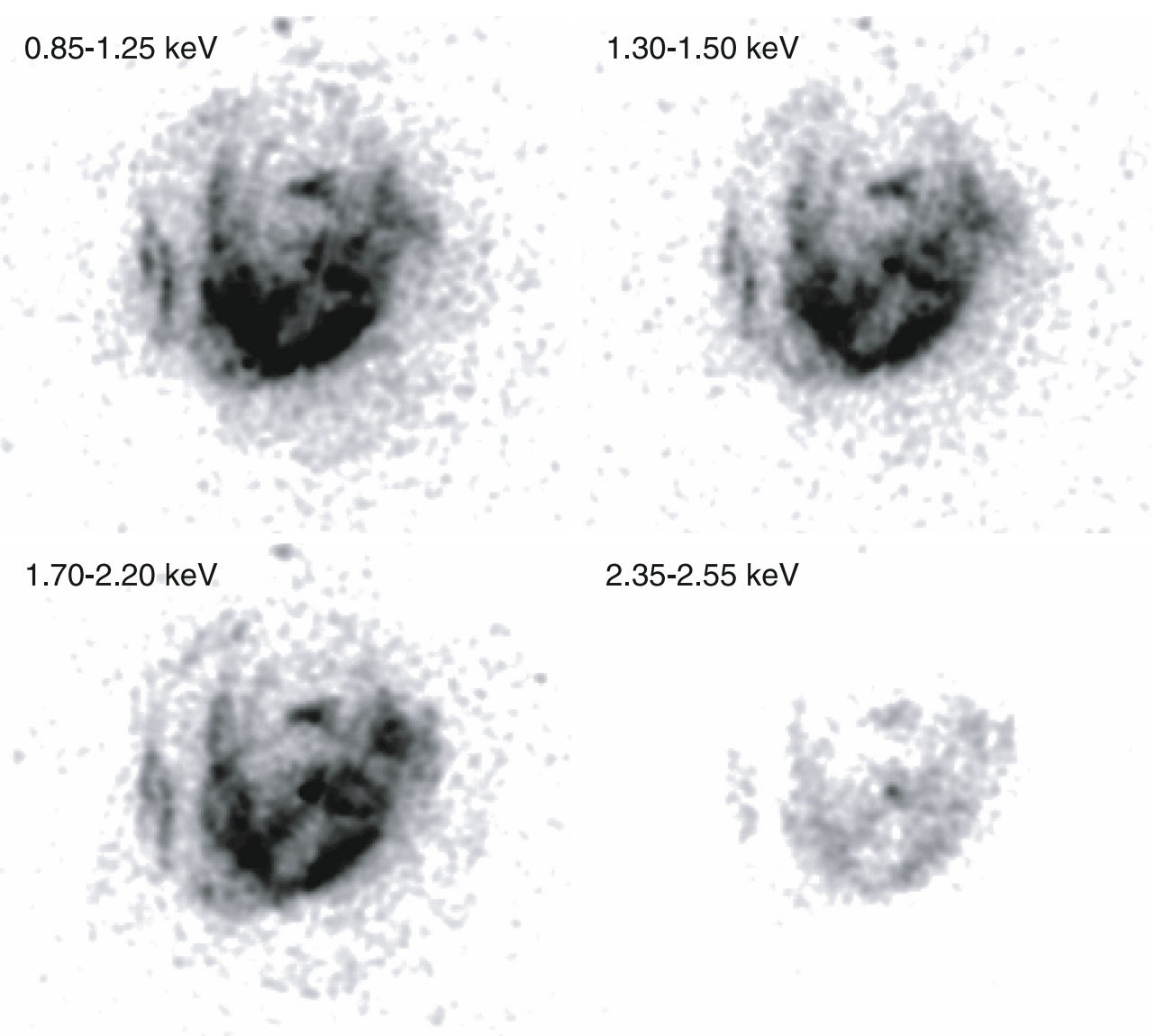

$2.35-2.55 \mathrm{keV}$

Fig. 7. XMM-Newton EPIC images of Kes 79 in the bands centered at: Ne line (0.85-1.25 keV), Mg line (1.30-1.50 keV), Si line (1.70-2.20 keV), and $\mathrm{S}$ line $(2.35-2.55 \mathrm{keV})$. The grayscale is the same for all images to provide a comparative view of the X-ray emission distribution.

whereas the Si emission in the south and southeast is weaker than that of either $\mathrm{Ne}$ or $\mathrm{Mg}$.

\subsection{X-ray spectroscopy}

MOS spectra of both observations (namely 0204970201 and 0204970301) were extracted from a circular region centered on $18^{\mathrm{h}} 52^{\mathrm{m}} 41^{\mathrm{s}},+00^{\circ} 40^{\prime} 47^{\prime \prime}$ of 4.74 radius, encompassing the complete SNR as observed in X-rays. The background spectra were obtained from four circular source-free regions of 0'73 radius located roughly to the north, east, south, and west of the remnant.

We fit the data from both observations simultaneously using a non-equilibrium ionization collisional plasma model (Borkowski et al. 2001), VNEI version 2.0, in XPSEC 11.3. 
Table 3. The best-fit of the global X-ray spectrum of Kes 79. The elemental abundances listed are those left free parameters in the model.

\begin{tabular}{lc}
\hline \hline Parameters & Values \\
\hline$k T(\mathrm{keV})$ & $0.70 \pm 0.01$ \\
$\tau\left(\mathrm{s} \mathrm{cm}^{-3}\right)$ & $6.2 \pm 0.2 \times 10^{10}$ \\
Ne abundance & $0.67 \pm 0.02$ \\
Mg abundance & $0.65 \pm 0.02$ \\
Si abundance & $0.66 \pm 0.01$ \\
S abundance & $1.25_{-0.06}^{+0.05}$ \\
Ar abundance & $5.0_{-1.6}^{+0.8}$ \\
Fe abundance & $0.54_{-0.02}^{+0.01}$ \\
$N_{\mathrm{H}}\left(\mathrm{cm}^{-2}\right)$ & $1.52_{-0.02}^{+0.01} \times 10^{22}$ \\
\hline
\end{tabular}

The absorption, temperature, ionization timescale, normalization, and abundances of $\mathrm{Ne}, \mathrm{Mg}, \mathrm{Si}, \mathrm{S}, \mathrm{Ar}$, and $\mathrm{Fe}$ are free parameters, whereas other elemental abundances are frozen to the solar values of Anders \& Grevesse (1989) after verifying that allowing them to vary does not significantly improve the model goodness-of-fit.

We obtained a statistically acceptable fit in the $0.5-5.0 \mathrm{keV}$ band (reduced $\chi^{2}$ of 1.33 for 991 degrees of freedom) with parameters values listed in Table 3 ; the spectra and data to model ratios are shown in Fig. 8. The best-fit requires lower than solar abundances of $\mathrm{Ne}, \mathrm{Mg}, \mathrm{Si}$, and $\mathrm{Fe}$, a slight overabundance of $\mathrm{S}$ and a somewhat larger overabundance of $\mathrm{Ar}$, although the last value is poorly constrained. Our results are consistent with those obtained from Chandra data by Sun et al. (2004) using the XSPEC VNEI plasma code (although the version is different), except for a $30 \%$ higher $\mathrm{Ne}$ abundance required for the $X M M$ Newton data.

\subsection{Discussion}

The new high resolution, low frequency radio data confirm the multi-shell morphology of Kes 79. One of the scenarios proposed to explain the presence of concentric shells in SNRs is that of a SN forward shock propagating within a wind bubble created by the massive precursor star. The existence of an X-ray pulsar in the interior of Kes 79 confirms that the SN precursor was a massive star that generated powerful winds during its lifetime. As summarized in Sect. 1, theoretical models predict in these cases transmitted and reflected shocks that originated in the interaction of the SN shock with the structural features left by the stellar wind. In this framework, the outer shell would represent the blast wave moving into the dense wall of the bubble, while the inner shell would represent the reflected shock travelling back towards the remnant's center.

Shocks of different origins should have different physical conditions (such as for example different compression ratios) and this should reflect in the synchrotron spectrum. From our detailed study, we found that the inner and outer shells have very similar spectral behavior, with a radio spectral index close to the global $\alpha \sim-0.6$. Thus, in terms of the radio spectrum, our study found that the shells are indistinguishable.

We can investigate the nature of the SN precursor based on the properties of the observed swept-up HI shell (Fig. 4). For this HI structure, we derived a radius of $17 \mathrm{pc}$ and a slow expansion velocity of $7 \mathrm{~km} \mathrm{~s}^{-1}$. Assuming a linear expansion regime to first approximation, we estimate its age to be about $2 \times 10^{6} \mathrm{yr}$. From the Chandra X-ray study, Sun et al. (2004) estimated that the age of Kes 79 is between $\sim 3000$ and $\sim 7800 \mathrm{yr}$, depending on

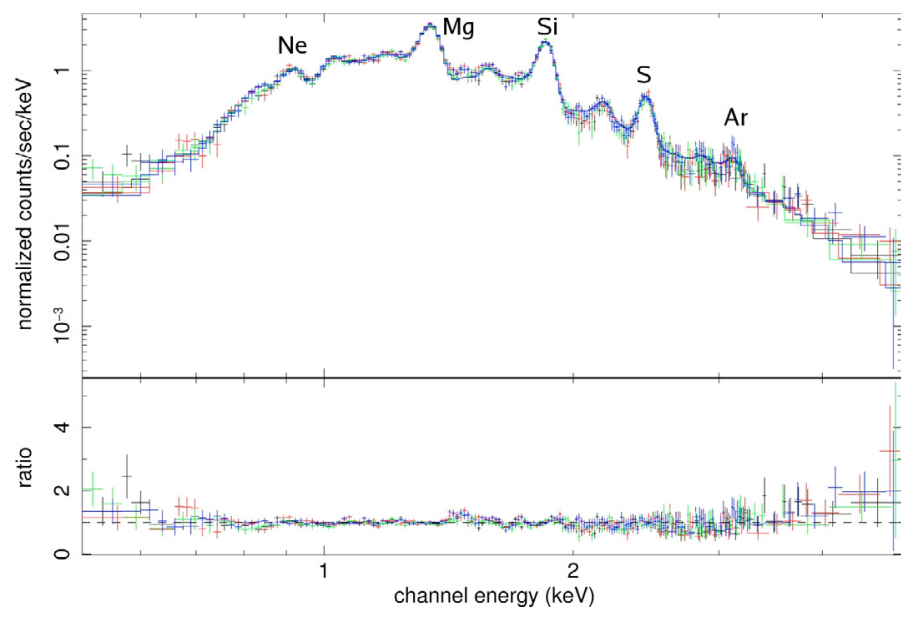

Fig. 8. EPIC spectra and data-to-model ratios of Kes 79 for observations 0204970201 (MOS1 black, MOS2 red) and 0204970301 (MOS1 green, MOS2 blue).

the assumed parameters (e.g., shock temperature, shock velocity, density contrast), while from radio data, we can set an upper limit to the age by applying Chevalier (1974)'s model, obtaining $t=\left(R_{\mathrm{pc}} / 21.9\right)^{3.23} \times 10^{5} \mathrm{yr} \simeq 15 \times 10^{3} \mathrm{yr}($ where $R \simeq 12.2 \mathrm{pc}$ is the assumed radius of the radio remnant). In any case, since the SNR is orders of magnitude younger than the HI shell, the detected cold neutral envelope must have formed during the earlier history of the star and must be a wind-driven shell. We can therefore confidently use the physical parameters of the associated HI shell to estimate the power of the stellar wind and hence infer the spectral type of the progenitor star. Wind blown HI-shells such as the one detected around Kes 79 have been observed around other massive OB and WR stars (e.g., Giacani \& Dubner 2004; Cappa et al. 2005; Cichowolski et al. 2008).

The kinetic energy of the HI shell structure can be compared with the mechanical energy released by a star into the ISM during the lifetime of the bubble, calculated with the equation $E_{\mathrm{w}}=10^{43} \dot{M} V_{\infty}^{2} t_{\mathrm{dyn}} / 2$, where $\dot{M}$ is the mass-loss rate of the star in $M_{\odot} / \mathrm{yr}, V_{\infty}$ is the terminal velocity of the wind in $\mathrm{km} \mathrm{s}^{-1}$, and $t_{\mathrm{dyn}}$ is the dynamical age of the structure in years. If we assume that only one star has powered the observed bubble, a single 09 star with a mass-loss rate of $\dot{M} \sim 10^{-7} M_{\odot} / \mathrm{yr}$ (Garmany et al. 1981) and a wind velocity of about $2000 \mathrm{~km} \mathrm{~s}^{-1}$ (Prinja et al. 1990) would be sufficient to sweep up the amount of interstellar gas observed to have accumulated in the shell, taking into account that the energy conversion efficiency in a bubble is no more than $20 \%$ (see Koo \& McKee 1992). In this case, the mechanical energy released by this star into the ISM is about $8 \times 10^{48} \mathrm{erg}$, enough to explain the calculated kinetic energy of the slowly expanding HI shell $\left(1.5 \times 10^{48} \mathrm{erg}\right)$.

The elemental abundances derived from the new X-ray spectroscopy, are in general, consistent with the results of Sun et al. (2004), suggesting that we are not observing shocked stellar ejecta but swept-up interstellar matter cooling and evaporating in the post-shock region. This picture is compatible with a scenario of a middle-age SNR evolving within a wind-driven bubble, where CSM and ISM have been crossed by forward and reverse shocks.

Based on the measured flux density at $324 \mathrm{MHz}$, we estimated the radio luminosity of Kes 79 to be $L_{\mathrm{R}}=2 \times 10^{34} \mathrm{erg} \mathrm{s}^{-1}$, for the assumed distance of $7 \mathrm{kpc}$ and the frequency range $10^{7}$ to $10^{11} \mathrm{~Hz}$. When compared to the X-ray luminosity derived for this SNR by Sun et al. (2004) between 0.5 and $10 \mathrm{keV}$, the 

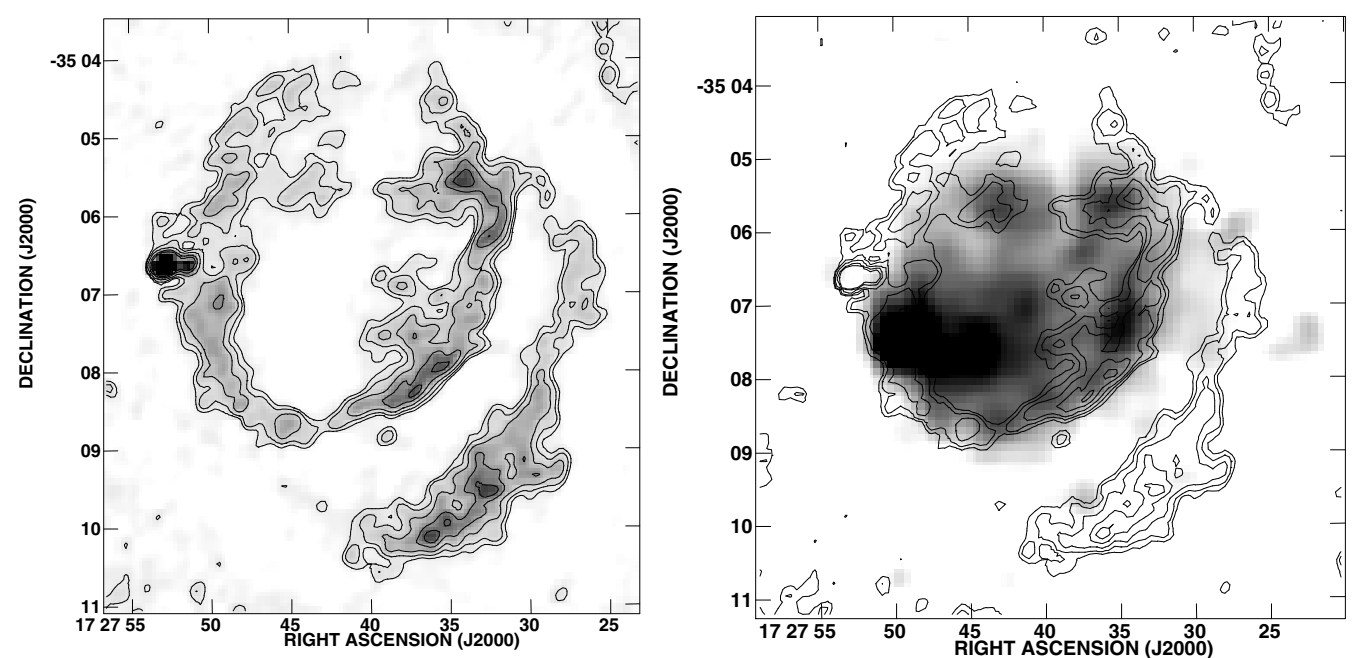

Fig. 9. Left: gray-scale and contour image of G352.7-0.1 at $4.8 \mathrm{GHz}$. The grayscale ranges from -0.1 to $3 \mathrm{mJy}^{\mathrm{beam}}{ }^{-1}$ and the contours level are: $0.15,0.3,0.6,1.2$, and $1.8 \mathrm{mJy}_{\text {beam }}^{-1}$. The beam size is $12^{\prime \prime} \times 9^{\prime \prime}$ and the noise is $0.2 \mathrm{mJy}$ beam ${ }^{-1}$. Right: XMM-Newton image in the $0.15-8 \mathrm{keV}$ energy band overlaid with radio contours at $4.8 \mathrm{GHz}$.

resulting ratio is over 100 . Such high ratios are common in relatively young SNRs evolving within or at the edges of a molecular cloud, as in the case of Kes 79. A large amount of the kinetic energy of a SNR can transform into bright emission X-rays, as observed for example in N132D in the LMC (Banas et al. 1997) and in the Galactic SNR G349.7+0.2 (Slane et al. 2002).

\section{Results for G352.7-0.1}

\subsection{Radio emission distribution}

The new VLA image of G352.7-0.1 at $4.8 \mathrm{GHz}$ is shown in the left panel of Fig. 9. The overall appearance of this image resembles that at $1.4 \mathrm{GHz}$ obtained by Dubner et al. (1993), but the higher sensitivity and angular resolution achieved in the $4.8 \mathrm{GHz}$ image indicate new internal emission, such as the features detected close to Dec $\sim-35^{\circ} 05^{\prime} 30^{\prime \prime}$ (which strikingly match two X-ray maxima, as can be seen in Fig. 9, right), as well as considerable small scale-features across the shells. It also resolves the bright spot detected by Dubner et al. (1993) at $1.4 \mathrm{GHz}$ at the eastern rim of G352.7-0.1 into the two point-like sources catalogued by White et al. (2005) as WBH2005 352.775-0.153, centered on $17^{\mathrm{h}} 27^{\mathrm{m}} 52^{\mathrm{s}} .8,-35^{\circ} 06^{\prime} 37^{\prime \prime}$, and WBH2005 352.772-0.149, centered on $17^{\mathrm{h}} 27^{\mathrm{m}} 51^{\mathrm{s}} .4,-35^{\circ} 06^{\prime} 37^{\prime \prime} 3$. As a control of the accuracy of the new $4.8 \mathrm{GHz}$ radio image, we estimated the flux density of these two radio point sources, obtaining $18 \mathrm{mJy}$ and $3.4 \mathrm{mJy}$ for WBH2005 352.775-0.153 and WBH2005 352.772-0.149, respectively, values that are in very good agreement with those of $18.07 \mathrm{mJy}$ and $3.34 \mathrm{mJy}$ published by White et al. (2005).

Because of their peculiar location overlapping the SNR radio shell, it is important to discern whether one or both of these point-like sources are part of the SNR structure or they are aligned by chance along the line of sight. To investigate this issue, we calculated their radio spectral index between 1.4 and $4.8 \mathrm{GHz}$ by carefully matching both images in all aspects (e.g., $u v$ coverage, center and pixel alignment). Since the two radio sources cannot be resolved in the lower resolution $1.4 \mathrm{GHz}$ image, the value of the spectral index is averaged across it. The flux density of the spot is $\sim 40 \mathrm{mJy}$ at $1.4 \mathrm{GHz}$ and $\sim 8.8 \mathrm{mJy}$ at $4.8 \mathrm{GHz}$. In both cases, the contribution of the shell emission has been subtracted. The resulting spectral index is $\alpha \sim-1.3$. To confirm this result, we also computed the spectral index using the T-T plot technique (Costain 1960; Turtle et al. 1962). This method is useful because it is unaffected by absolute calibration and offset variations between the images. With this method, the spectral index is $\alpha \sim-1.1$. Although the angular resolution of the available data does not allow us to estimate the spectral index for the two point-like sources separately, the obtained value suggests that these radio sources are extragalactic objects superimposed by chance along the line of sight. Higher angular resolution observations will help us to discern whether the peculiar double source appearance can be explained as having originated in clouds of radio-emitting plasma that were ejected by a distant active galactic nuclei (AGN) in narrow jets (the type of double-lobed sources called "DRAGN", Double Radiosource Associated with Galactic Nucleus). For the present study of G352.7-0.1, the main conclusion is that the sources are not related to the SNR.

\subsection{The distance to G352.7-0.1}

We investigated the distribution of the interstellar gas in the direction of G352.7-0.1, with the hope of finding some morphological concordance between the SNR and the surrounding matter that would help us to constrain its systemic radial velocity. The HI distribution was inspected by analyzing data extracted from the Southern Galactic Plane Survey obtained with the Australia Telescope Compact Array and Parkes Radiotelescopes (McClure-Griffiths et al. 2005). The angular resolution of the survey is about $2^{\prime}$ and the velocity resolution, $0.82 \mathrm{~km} \mathrm{~s}^{-1}$. The numerous contributions of $\mathrm{HI}$ emission in this direction of the Galaxy, close to the Galactic center and above the Galactic plane, however, impeded the unambiguous identification of associated gaseous structures. We therefore limited our study to HI absorption.

HI absorption profiles were traced in the direction of bright emission radio continuum regions in G352.7-0.1, where we found that the most distant HI absorption feature towards G352.7-0.1 appears close to the radial velocity $v_{\mathrm{LSR}}=$ $-90 \mathrm{~km} \mathrm{~s}^{-1}$. By applying the Galactic circular rotation model of Fich et al. (1989), this LSR velocity corresponds to the near and far distances of $\sim 6.8$ or $\sim 10.1 \mathrm{kpc}$. We can safely assume that $6.8 \mathrm{kpc}$ is the lower limit to the distance to G352.7-0.1. The 


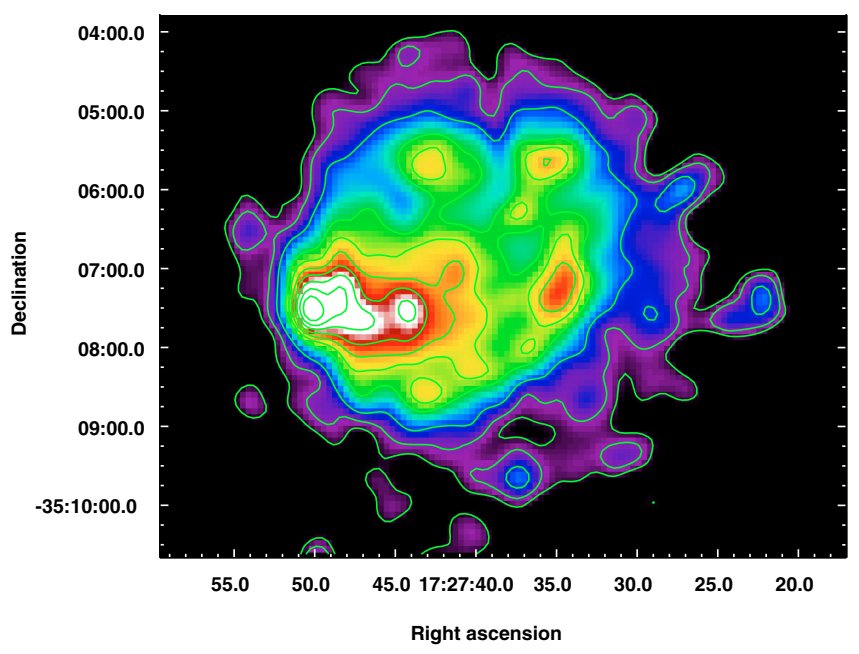

Fig. 10. XMM-Newton EPIC image of G352.7-0.1 in the broadband $0.15-0.8 \mathrm{keV}$ energy band.

upper limit to the distance can be determined from the Galactic tangent point velocity, $v_{\mathrm{LSR}} \sim-190 \mathrm{~km} \mathrm{~s}^{-1}$, which corresponds approximately to a kinematical distance of $8.4 \mathrm{kpc}$. Thus, the distance to G352.7-0.1 is in between $\sim 6.8$ and $\sim 8.4 \mathrm{kpc}$. In a modern 2-armed spiral pattern of the inner galaxy (e.g., Dame \& Thaddeus 2008; Englmaier et al. 2008), if G352.7-0.1 is located at $6.8 \mathrm{kpc}$, it would lie over the "near $3 \mathrm{kpc}$ arm", while at $8.4 \mathrm{kpc}$ it would be part of the central bar. Since both of the obtained limits are plausible, we conclude that a value of $7.5 \pm 0.5 \mathrm{kpc}$ is an adequate estimate of the distance to G352.7-0.1.

\subsection{Analysis of $X$-ray emission and comparison with radio}

Figure 10 displays the broadband (0.15-0.8 keV) XMM-Newton EPIC image of the SNR G352.7-0.1. The EPIC, PN and MOS images were merged and smoothed with a $8^{\prime \prime}$ (FWHM) Gaussian. This new high angular resolution image shows considerable clumpy structures and diffuse emission filling the interior of the remnant. The outermost and faintest X-ray emission traces an almost circular boundary about $6^{\prime}$ in diameter. Most of the X-ray emission is concentrated in an elongated feature, whose strongest X-ray peak lies in the eastern border of the remnant, centered on about $17^{\mathrm{h}} 27^{\mathrm{m}} 50^{\mathrm{s}} .2,-35^{\circ} 07^{\prime} 28^{\prime \prime} 8$. Three remarkable bright knots appear at the north and west border of G352.7-0.1 centered on about: $17^{\mathrm{h}} 27^{\mathrm{m}} 43^{\mathrm{s}},-35^{\circ} 05^{\prime} 37^{\prime \prime}$; $17^{\mathrm{h}} 27^{\mathrm{m}} 35^{\mathrm{s}} .6,-35^{\circ} 05^{\prime} 38^{\prime \prime} 5$; and $17^{\mathrm{h}} 27^{\mathrm{m}} 35^{\mathrm{s}} .9,-35^{\circ} 07^{\prime} 15^{\prime \prime} 7$.

Narrow-band images centered on the Si (1.7-2 keV), S (2.3-2.6 keV), Ar (3.1-3.3 keV), and Fe (6.3-6.7 keV) emission are displayed in Fig. 11. The same grayscale is used for all the images to provide a comparative view of the X-ray emission distribution. In contrast, the contour levels vary from an image to the other to emphasize the most significant features in each one. From this figure, can be inferred that soft X-rays dominate the emission. The distribution of the emission in the energy bands centered on the Si and S lines does not show appreciable differences from the broadband image, while the image centered on the Ar line, instead, is remarkable only in the elongated brightest X-ray feature in the southeast. The distribution of the Fe emission line band is more clumpy and anticorrelated with the other bands, except for the northern knot centered on $17^{\mathrm{h}} 27^{\mathrm{m}} 43^{\mathrm{s}},-35^{\circ} 05^{\prime} 37^{\prime \prime}$, which is the only X-ray feature detected in all four X-ray bands.
Using our high-resolution radio and X-ray images, we are able to compare for the first time both morphologies in great detail. Figure 9 (right) shows a grayscale XMM-Newton image with some radio contours at $4.8 \mathrm{GHz}$ superimposed. The improved $\mathrm{X}$-ray image shows that $\mathrm{X}$-ray emission is confined to within the inner radio shell, filling it completely. In general, there is no positional correspondence between the radio synchrotron and the thermal X-ray emitting plasmas, except for the already noted correspondence near Dec $-35^{\circ} 05^{\prime} 30^{\prime \prime}$, where the bright X-ray spots coincide with the two radio maxima detected in the interior of the radio shell, while the brightest X-ray features, close to $17^{\mathrm{h}} 27^{\mathrm{m}} 50^{\mathrm{s}},-35^{\circ} 07^{\prime} 30^{\prime \prime}$, lack any conspicuous radio counterpart. The eastern radio point sources, WBH2005 352.775-0.153 and WBH2005 352.772-0.149, do not have any X-ray counterpart, confirming that they are unrelated to the SNR.

\subsection{X-ray spectroscopy}

We extracted the MOS1, MOS2, and PN spectra from a circular region of radius 2.5 centered on $17^{\mathrm{h}} 27^{\mathrm{m}} 36^{\mathrm{s}},-35^{\circ} 06^{\prime} 55^{\prime \prime}$, which covers the entire X-ray emission observed. The background spectrum for the three instruments was taken from an annular source free region around the remnant with inner and outer radii of 4.0 and 4.7, respectively. G352.7-0.1 is located in the Galactic ridge region, known to have enhanced thin thermal emission. However, as discussed by Kinugasa et al. (1998), variations in the background with latitude and longitude in this region, can be neglected.

The data were simultaneously fit in the $0.7-7.5 \mathrm{keV}$ energy band with a non-equilibrium ionization collisional plasma model (Borkowski et al. 2001), assuming a constant temperature and single ionization parameter, combined with interstellar absorption. The absorption, plasma temperature, ionization timescale, normalization, and abundances of $\mathrm{S}, \mathrm{Si}$, and Ar were considered to be free parameters in the model. We verified that allowing other elemental abundances to vary did not significantly improve the model fit, hence there were frozen to the solar values of Anders \& Grevesse (1989). An additional zero-width Gaussian component was needed to model the $\mathrm{Fe} \mathrm{K}$ emission resulting in a line centroid at $6.46 \pm 0.03 \mathrm{keV}$, which would be consistent with emission caused by $\mathrm{Fe} \mathrm{K} \alpha$ fluorescence. We obtained a statistically good fit (with a reduced $\chi^{2}$ of 1.25 for 594 degrees of freedom), the results of which are listed in Table 4 and plotted in Fig. 12. As can be seen, the best fit requires significant over abundances of S, Si, and Ar with respect to their solar values, which indicates the presence of ejecta material. The Fe L abundance does not differ significantly from the solar value, which argues in favor of a core collapse SN. However, this cannot fully constrain the progenitor explosion because the relative lack of $\mathrm{Fe}$ could only be explained if the innermost ejecta layers have not yet been shocked (Rakowski et al. 2006). Regarding the Fe K line at an energy of $6.46 \mathrm{keV}$, it has also been observed in other young SNRs such as Tycho (Hwang \& Gotthelf 1997), Kepler (Kinugasa \& Tsunemi 1999), and RCW86 (Bamba et al. 2000), and also in G344.7-0.1 (Yamauchi et al. 2005). The origin of this line is not well understood.

We searched for spectral variations across the remnant by individually extracting the spectrum of each of the most conspicuous X-ray features and the diffuse emission. This procedure was performed separately for each instrument. We found no significant difference in the fit parameters for any of them compared with the global model.

Based on the emission measure (EM) determined from our spectral fitting and a distance of $7.5 \mathrm{kpc}$, we can estimate the 

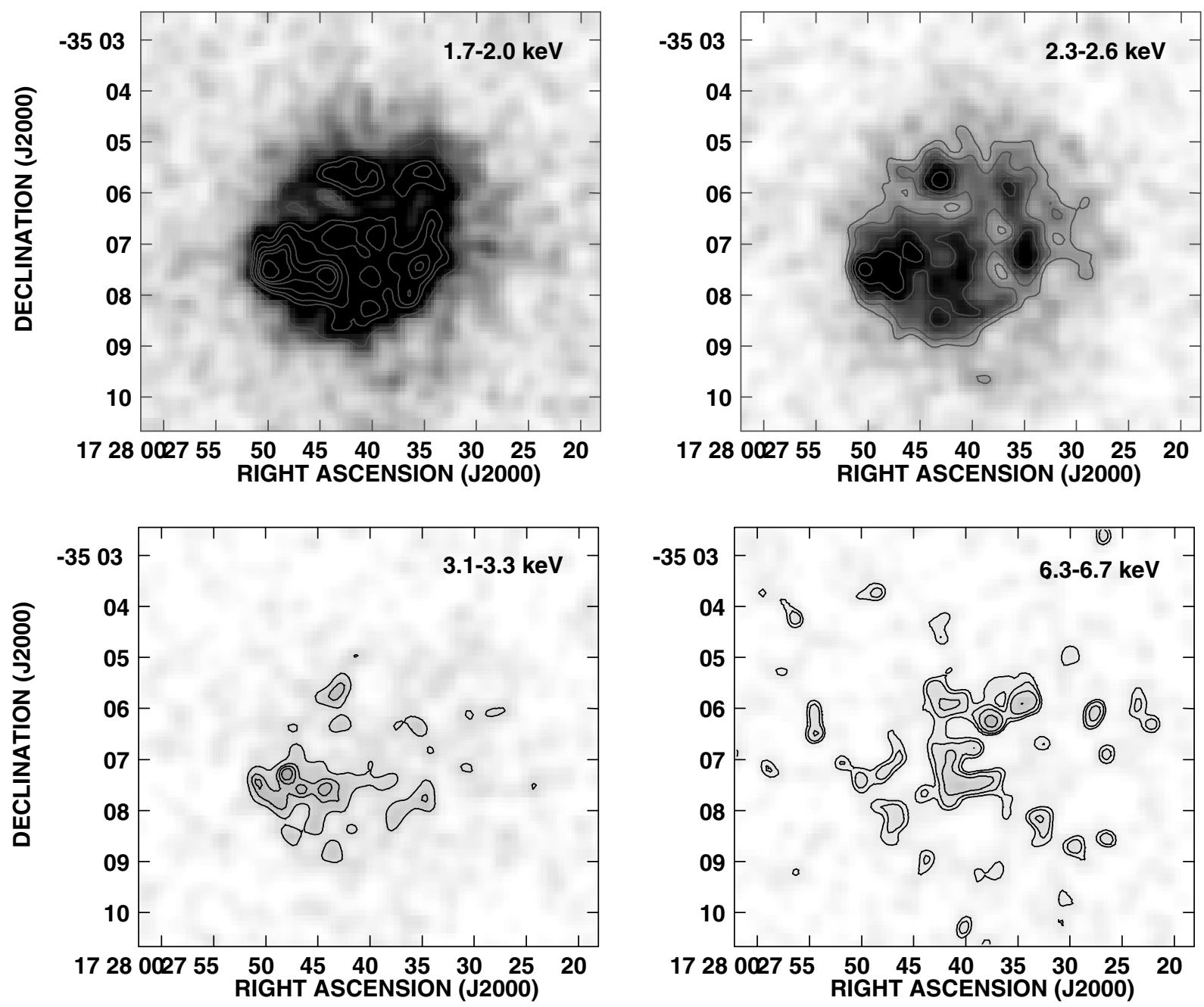

Fig. 11. XMM-Newton EPIC images of G352.7-0.1 in the bands centered at: Si XIII/XV line (1.7-2 keV), S XIII/XV line (2.3-2.6 keV), Ar XVII line $(3.1-3.3 \mathrm{keV})$ and $\mathrm{Fe} \mathrm{K} \alpha$ line $(6.3-6.7 \mathrm{keV})$. The grayscale is the same for all images to provide a comparative view of the X-ray emission distribution. The contours level varies from an image to the other to remark the most significant features in each one of them.

Table 4. The best-fit model of the global X-ray spectrum of G352.7-0.1. The elemental abundances listed are those considered to be free parameters of the model.

\begin{tabular}{lc}
\hline \hline Parameters & Values \\
\hline$k T(\mathrm{keV})$ & $1.9 \pm 0.2$ \\
$\tau\left(\mathrm{s} \mathrm{cm}^{-3}\right)$ & $4.5 \pm 0.5 \times 10^{10}$ \\
Si abundance & $2.4 \pm 0.2$ \\
S abundance & $3.8 \pm 0.3$ \\
Ar abundance & $4.7 \pm 1.2$ \\
$N_{\mathrm{H}}\left(\mathrm{cm}^{-2}\right)$ & $2.6 \pm 0.3 \times 10^{22}$ \\
\hline
\end{tabular}

electron density of the plasma to be $n_{\mathrm{e}}=(E M / V)^{1 / 2} \simeq 0.3 \mathrm{~cm}^{-3}$, where $V$ is the volume of the X-ray emitting plasma considered in this case to be a sphere of 2.5 of radius, and the hydrogen number density, $n_{\mathrm{e}}=n_{\mathrm{H}}$, for simplicity. The age of the remnant, estimated to be $\tau / n_{\mathrm{e}}$, is found to be $4700 \mathrm{yr}$, and the mass of the X-ray emitting gas is $M=n_{\mathrm{e}} V m_{\mathrm{H}} \simeq 10 M_{\odot}$, where $m_{\mathrm{H}}$ is the hydrogen atomic mass. For a shock temperature of $k T=1.9 \mathrm{keV}$, the shock velocity would be $v_{\mathrm{s}}=$ $\left(16 k T / 3 \mu m_{\mathrm{H}}\right)^{1 / 2} \simeq 1300 \mathrm{~km} \mathrm{~s}^{-1}$ (with a mean atomic weight $\mu=0.61$ ). For the preceding numerical values, we estimated the supernova explosion energy $\mathrm{E}$ to be about $10^{50} \mathrm{erg}$, which is

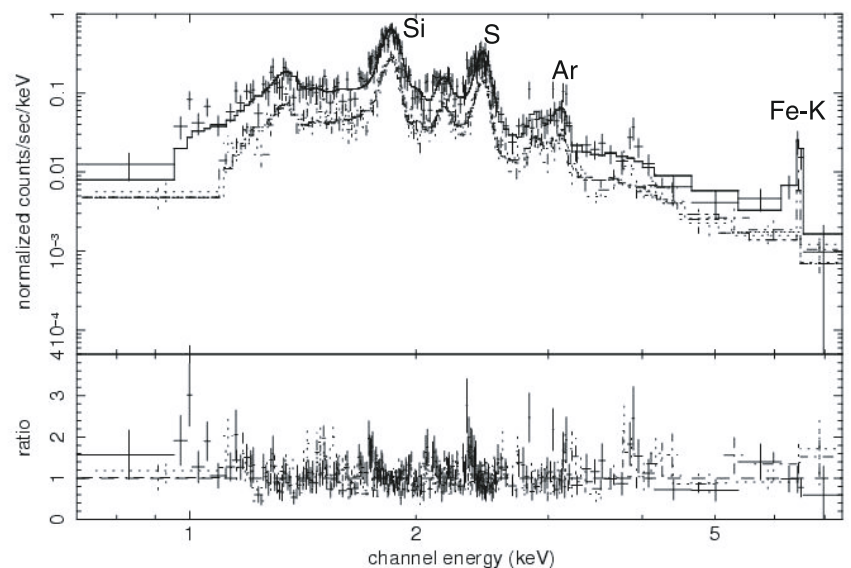

Fig. 12. Energy spectra of G352.7-0.1. The lines for different instruments are: solid for PN, dash-dotted for MOS1, and dotted for MOS2.

the standard value for SN explosions. Based on the NEI fit to the spectrum of the entire remnant in the (0.7-7.5) keV energy band, we estimate the X-ray luminosity to be $L_{\mathrm{X}} \sim 8.4 \times 10^{34} \mathrm{erg} \mathrm{s}^{-1}$ 


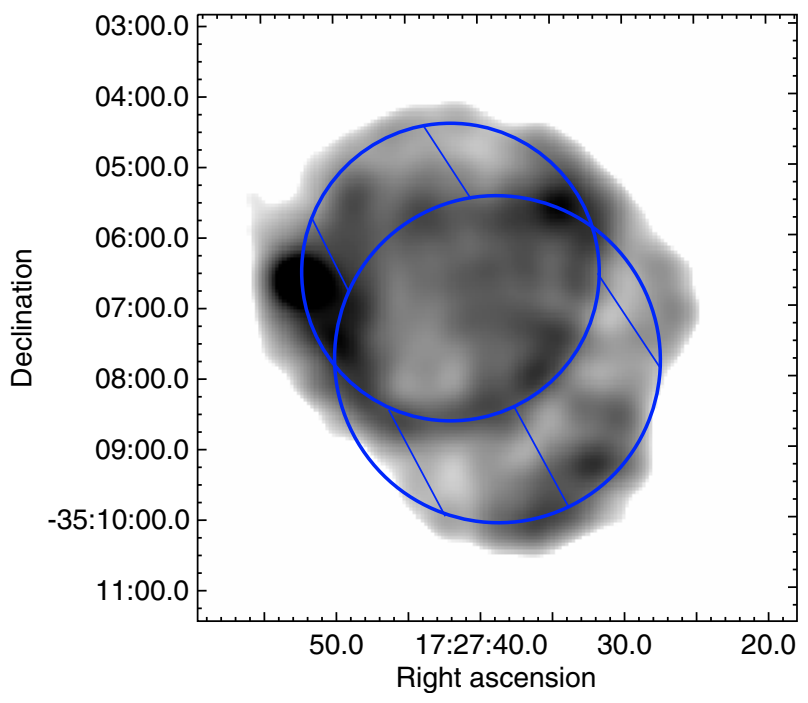

Fig. 13. Model of "barrel-shaped" SNR overlapping the VLA image of G352.7-0.1 at $1.4 \mathrm{GHz}$ from Dubner et al. (1993).

for a distance of $7.5 \mathrm{kpc}$. This value can be compared with the radio luminosity calculated on the basis of the flux density measurement at $1465 \mathrm{MHz}$ carried out by Dubner et al. (1993) to obtain $L_{\mathrm{R}}=4.6 \times 10^{33} \mathrm{erg} \mathrm{s}^{-1}$, and a ratio $L_{\mathrm{X}} / L_{\mathrm{R}} \sim 20$.

\subsection{Discussion}

Kinugasa et al. (1998) described the X-ray emission of G3527-0.1 as shell-type with a ring morphology similar to that observed in the radio band. However, our new sensitive XMMNewton image of this SNR indicates that the X-ray emission completely fills the interior of the radio remnant. Although the centroid of the X-ray emission lies over the eastern half of the remnant, both the observed morphology and the thermal nature of the emission with a flat radial temperature profile, allow us to re-classify G352.7-0.1 as a mixed-morphology remnant, i.e., shell-type in radio and filled-center in X-rays (Rho \& Petre 1998). The X-ray emitting plasma has not yet reached the ionization equilibrium, as expected for young remnants, and has enhanced metal abundances, indicative of an ejecta origin.

The observed morphology matches a "barrel-shaped" model fairly well for the radio SNR (that is, a structure with cylindrical geometry in space, as discussed by Manchester 1987; and Gaensler 1998) with a peculiar viewing angle as depicted in Fig. 13. To produce this figure we used the VLA image at 1.4 GHz from Dubner et al. (1993), which recovered all the flux density. The radio emission appears to be enhanced in the directions where our line of sight crosses a longer path through the SNR. As proposed by Manchester (1987), the bilateral enhancements observed in "barrel-shaped" SNRs can originate in a biconical flow from the pre-supernova star, from the supernova explosion itself, or from an associated pulsar or X-ray binary system, and is probably formed early in the life of the remnant. In the case of G352.7-0.1, which is known not to harbor a pulsar in its interior, the origin of the observed appearance must be connected with the mass-loss history of the progenitor star. In this context, Manchester (1987) proposed that a biannular or double ring morphology can be produced when the SN shock expands through the axially symmetric wind of a red supergigant (RSG) phase of a massive star. Typical parameters for the RSG phase are a wind velocity of about $20 \mathrm{~km} \mathrm{~s}^{-1}$ and a lifetime of about
$3 \times 10^{5} \mathrm{yr}$ (Smith 1994). This wind perturbs a region with a radius of $6 \mathrm{pc}$, a size comparable to the radius of G352.7-0.1. The $\mathrm{X}$-ray spectrum results, which favor a type II explosion for this $\mathrm{SN}$, confirm a scenario that involves a massive star precursor.

\section{Conclusions}

We have analyzed new high resolution and sensitivity VLA images as well as archival radio and XMM-Newton X-ray data of two galactic SNRs, Kes 79 and G352.7-0.1, which share a common multi-shell radio morphology. In addition, we have investigated the ISM in the direction of both remnants to probe the conditions of their surroundings.

New radio features detected in the image of Kes 79 at $324 \mathrm{MHz}$ considerably improve the correspondence with the $\mathrm{X}$-ray emission distribution of this remnant. Based on our new radio data and flux density estimates taken from the literature, we derived a global spectral index of $\alpha=-0.58 \pm 0.03$, constant over four decades in frequency with no significant turnover down to $74 \mathrm{MHz}$. The new 74 and $324 \mathrm{MHz}$ data and the reprocessed $1500 \mathrm{MHz}$ data were combined to perform the first careful, spatially resolved study of the synchrotron spectrum in Kes 79 , concluding that the radio spectrum is homogeneous, with no detectable variations associated with shells, maxima, or any other morphological feature. We have also confirmed that down to about $5.8 \mathrm{mJy} \mathrm{beam}^{-1}$, neither a radio counterpart to the X-ray pulsar CXOU J185238.6+004020, nor a surrounding PWN is observed. Our study confirms that Kes 79 is the result of a corecollapse SNe evolving close to a molecular cloud and within the wind-blown bubble created by its precursor. The average Xray spectral properties are well described by a non-equilibrium ionization collisional plasma model with constant temperature across the remnant and solar abundances. The observed multishell appearance of Kes 79 can be described as the final product of possibly a $\mathrm{O} 9$ star exploding within the cavity created by previous episodes of mass loss and evolving near the parent molecular cloud. The precursor wind has swept up the surrounding gas forming a thick HI shell. A scenario in which the multishell morphology originated from multiple shocks produced after the encounter of the blast wave with a density jump in the surroundings, could explain the observations.

In the case of the SNR G352.7-0.1, the new VLA image at $4.8 \mathrm{GHz}$ uncovered considerable clumpy structures on small scales, confirming that the bright spot previously observed on the eastern limb is produced by a double extragalactic radio source. The new XMM-Newton image shows several knots of emission and diffuse thermal emission filling the interior of the remnant. The X-ray spectral investigation inferred enhanced abundances, which imply the presence of SN ejecta in this SNR. From the morphology and spectral properties of G352.7-0.1, we classify this remnant as belonging to the mixed-morphology category. The observed appearance can be the consequence of the propagation of the SN blast-wave within an axially symmetric stellar wind blown out by the precursor star, forming a "barrel-shaped" SNR that is observed at a peculiar viewing angle.

The study of the surrounding interstellar gas around both SNRs allowed us to refine the distance estimate towards these two Galactic SNRs, obtaining a distance of $7 \pm 0.5 \mathrm{kpc}$ for Kes 79 , and $7.5 \pm 0.5 \mathrm{kpc}$ for G352.7-0.1. Table 5 summarizes the main findings of the present study.

Acknowledgements. We thanks the anonymous referee for helpful comments. This research was partially funded by Argentina Grants awarded by ANPCYT, CONICET and University of Buenos Aires (UBACYT A023). This work is based on observations done with the XMM-Newton, an ESA science mission 
Table 5. Main results.

\begin{tabular}{|c|c|c|}
\hline & Kes 79 & G352.7-0.1 \\
\hline Radio flux density & $\begin{array}{l}\text { at } 74 \mathrm{MHz}=76 \mathrm{Jy} \\
\text { at } 324 \mathrm{MHz}=39 \mathrm{Jy} \\
\text { at } 1.5 \mathrm{GHz}=11 \mathrm{Jy}\end{array}$ & \\
\hline Radio spectral index & -0.58 & \\
\hline Distance & $7 \mathrm{kpc}$ & $7.5 \mathrm{kpc}$ \\
\hline Age & $<15000 \mathrm{yr}$ & $5000 \mathrm{yr}$ \\
\hline X-ray-to-Radio Luminosity & $\sim 100$ & $\sim 20$ \\
\hline Elemental abundances & Shocked CSM and ISM & Ejecta \\
\hline Scenario & $\begin{array}{l}\text { Core collapse of an } \mathrm{O} 9 \text { star } \\
\text { within a wind-driven bubble } \\
\text { plus projection }\end{array}$ & $\begin{array}{l}\text { Interaction with an asymmetric progenitor wind } \\
\text { resulting in a "barrel-shaped" SNR } \\
\text { plus viewing angle and projection }\end{array}$ \\
\hline
\end{tabular}

with instruments and contributions directly funded by ESA Member States and the US (NASA). The Canadian Galactic Plane Survey is a Canadian project with international partners, and is supported by the Natural Sciences and Engineering Research Council (NSERC). NL and MS are thankful to the companies INSA and Selex I. S. resp. for a financial support for the publication of this paper.

\section{References}

Altenhoff, W. J., Downes, D., Goad, L., Maxwell, A., \& Rinehart, R. 1970, A\&AS, 1,319

Anders, E., \& Grevesse, N. 1989, Geochim. Cosmochim. Acta, 53, 197

Baars, J. W. M., Genzel, R., Pauliny-Toth, I. I. K., \& Witzel, A. 1977, A\&A, 61, 99

Bamba, A., Koyama, K., \& Tomida, H. 2000, PASJ, 52, 1157

Banas, K. R., Hughes, J. P., Bronfman, L., \& Nyman, L.-A. 1997, ApJ, 480, 607

Beard, M., \& Kerr, F. J. 1969, Aust. J. Phys., 22, 121

Becker, R. H., \& Kundu, M. R. 1975, AJ, 80, 679

Borkowski, K., Szymkowiak, A. E., Blondin, J. M., \& Sarazin, C. L. 1996, ApJ, 466, 866

Borkowski, K. J., Lyerly, W. J., \& Reynolds, S. P. 2001, ApJ, 548, 820

Cappa, C., Niemela, V. S., Martín, M. C., \& McClure-Griffiths, N. M. 2005, A\&A, 436, 155

Case, G. L., \& Bhattacharya, D. 1998, ApJ, 504, 761

Castelletti, G., Dubner, G., Brogan, C., \& Kassim, N. E. 2007, A\&A, 471, 537

Caswell, J. L., Murray, J. D., Roger, R. S., Cole, D. J., \& Cooke, D. J. 1975, A\&A, 45, 239

Caswell, J. L., Milne, D. K., \& Wellington, K. J. 1981, MNRAS, 195, 89

Chevalier, R. A. 1974, ApJ, 188, 501

Cichowolski, S., Pineault, S., Arnal, E. M., \& Cappa, C. E. 2008, A\&A, 478, 443

Costain, C. H. 1960, MNRAS, 120, 248

Dame, T. M., \& Thaddeus, P. 2008, ApJ, 683, L143

Dickel, J. R. 1973, Aust. J. Phys., 26, 369

Dickman, R. L. 1978, ApJS, 37, 407

Dubner, G. M., Moffett, D. A., Goss, W. M., \& Winkler, P. F. 1993, AJ, 105, 2251

Dulk, P., \& Slee, O. 1972, Aust. J. Phys., 25, 429

Dwarkadas, V. V. 2005, ApJ, 630, 892

Englmaier, P., Pohl, M., \& Bissantz, N. 2008, ArXiv e-prints

Fich, M., Blitz, L., \& Stark, A. A. 1989, ApJ, 342, 272

Franco, J., Tenorio-Tagle, G., Bodenheimer, P., \& Rozyczka, M. 1991, PASP, 103,803

Gaensler, B. M. 1998, ApJ, 493, 781

Gaensler, B. M., \& Slane, P. O. 2006, ARA\&A, 44, 17

Garmany, C. D., Olson, G. L., van Steenberg, M. E., \& Conti, P. S. 1981, ApJ, 250,660
Giacani, E., \& Dubner, G. 2004, A\&A, 413, 225

Gotthelf, E. V., Halpern, J. P., \& Seward, F. D. 2005, ApJ, 627, 390

Green, D. A. 1989, MNRAS, 238, 737

Green, D. A. 2009, VizieR Online Data Catalog, 7253, 0

Green, D. A., \& Dewdney, P. E. 1992, MNRAS, 254, 686

Gvaramadze, V. V. 2006, The Ultraviolet Universe: Stars from Birth to Death,

26th meeting of the IAU, Joint Discussion 4, 16, 17 August 2006, Prague,

Czech Republic, JD04, \#2, 4

Halpern, J. P., Gotthelf, E. V., Camilo, F., \& Seward, F. D. 2007, ApJ, 665, 1304

Hwang, U., \& Gotthelf, E. V. 1997, ApJ, 475, 665

Jackson, J. M., Rathborne, J. M., Shah, R. Y., et al. 2006, ApJS, 163, 145

Kassim, N. E. 1989, ApJS, 71, 799

Kassim, N. E. 1992, AJ, 103, 943

Kesteven, M. J. L. 1968, Aust. J. Phys., 21, 369

Kinugasa, K., \& Tsunemi, H. 1999, PASJ, 51, 239

Kinugasa, K., Torii, K., Tsunemi, H., et al. 1998, PASJ, 50, 249

Koo, B.-C., \& McKee, C. F. 1992, ApJ, 388, 93

Kovalenko, A. V., Pynzar', A. V., \& Udal'Tsov, V. A. 1994, Astron. Rep., 38, 784

Kundu, M. R., \& Velusamy, T. 1967, Annales d'Astrophysique, 30, 723

Manchester, R. N. 1987, A\&A, 171, 205

McClure-Griffiths, N. M., Dickey, J. M., Gaensler, B. M., et al. 2005, ApJS, 158, 178

Prinja, R. K., Barlow, M. J., \& Howarth, I. D. 1990, ApJ, 361, 607

Rakowski, C. E., Badenes, C., Gaensler, B. M., et al. 2006, ApJ, 646, 982

Reich, W., Reich, P., \& Fuerst, E. 1990, A\&AS, 83, 539

Reynoso, E. M., Goss, W. M., Dubner, G. M., Winkler, P. F., \& Schwarz, U. J. 1997, A\&A, 317, 203

Rho, J., \& Petre, R. 1998, ApJ, 503, L167

Scoville, N. Z., Yun, M. S., Sanders, D. B., Clemens, D. P., \& Waller, W. H. 1987, ApJS, 63, 821

Seward, F. D., Slane, P. O., Smith, R. K., \& Sun, M. 2003, ApJ, 584, 414

Slane, P., Chen, Y., Lazendic, J. S., \& Hughes, J. P. 2002, ApJ, 580, 904

Slee, O. B. 1977, Aust. J. Phys. Astrophys. Supp., 43, 1

Smith, L. J. 1994, in Circumstellar Media in Late Stages of Stellar Evolution, ed. R. E. S. Clegg, I. R. Stevens, \& W. P. S. Meikle, 64

Sun, M., Seward, F. D., Smith, R. K., \& Slane, P. O. 2004, ApJ, 605, 742

Taylor, A. R., Gibson, S. J., Peracaula, M., et al. 2003, AJ, 125, 3145

Turtle, A. J., Pugh, J. F., Kenderdine, S., \& Pauliny-Toth, I. I. K. 1962, MNRAS, 124, 297

Uyanıker, B., Reich, W., Yar, A., Kothes, R., \& Fürst, E. 2002, A\&A, 389, L61 Velázquez, P. F., de la Fuente, E., Rosado, M., \& Raga, A. C. 2001, A\&A, 377, 1136

Velusamy, T., Becker, R. H., \& Seward, F. D. 1991, AJ, 102, 676

White, R. L., Becker, R. H., \& Helfand, D. J. 2005, AJ, 130, 586

Wilner, D. J., Reynolds, S. P., \& Moffett, D. A. 1998, AJ, 115, 247

Yamauchi, S., Ueno, M., Koyama, K., \& Bamba, A. 2005, PASJ, 57, 459 